\title{
Artenschutz und Europarecht im Kontext der Windenergie - Der Klimaschutz und die Auslegung der Ausnahmeregelungen der Vogelschutzrichtlinie
}

\author{
Ekkehard Hofmann*
}

(C) Der/die Autor(en) 2021. Dieser Artikel ist eine Open-Access-Publikation.

Während die FFH-Richtlinie ein flexibles und durch die Rechtsprechung in vielen Einzelfragen geklärtes Instrumentarium zur Rechtfertigung der Verletzung naturschutzrechtlicher Schutzvorschriften enthält, werden die entsprechenden Ausnahmevorschriften der Vogelschutzrichtlinie von Vielen als erheblich rigider empfunden. Das VG Gießen ging jüngst sogar soweit, die Erteilung einer Genehmigung für eine Windenergieanlage aufzuheben, weil die Anlage trotz aller Vorkehrungen zum Schutz der Vögel deren Tötungsrisiko signifikant erhöht hätte. Das gibt Anlass, der Frage nachzugehen, ob und inwieweit das Artenschutzrecht dem Ausbau der Windenergie tatsächlich substanzielle und möglicherweise einschneidende Grenzen setzt. Der Beitrag gelangt zu dem Ergebnis, dass die Vogelschutzrichtlinie bei näherer Analyse durchaus Möglichkeiten bietet, eine praktische Konkordanz der gegenläufigen Zielsetzungen zu erreichen.

\section{Einleitung: Die Auslegung artenschutzrechtlicher} Tötungsverbote als Schlüsselfrage der Energiewende

Spätestens seit dem Pariser Übereinkommen aus dem Jahr 2015 ist naturwissenschaftlich, politisch wie rechtlich anerkannt, dass nur eine Begrenzung der Erderwärmung auf höchstens 1,5 Grad Celsius gegenüber der vorindustriellen Zeit in der Lage sein wird, dramatische Veränderungen des Weltklimas noch zu verhindern. ${ }^{1}$ Gleichzeitig verdichten sich die Befürchtungen, dass auch hinsichtlich der Biodiversität die planetaren Belastungsgrenzen bald erreicht sein könnten. ${ }^{2}$ Während sich Maßnahmen des Klimaschutzes und solche des Artenschutzes sicherlich vielfach kohärent und in gegenseitiger Verstärkung ausgestalten lassen, ${ }^{3}$ können Konflikte trotz aller Vermeidungsmaßnahmen beim Ausbau der Wasserkraft, gerade aber auch dem der Windenergie nicht immer vollständig ausgeschlossen werden, so dass am Ende entschieden werden muss, welchem Interesse der Vorrang gebührt.

Diese Dichotomie ist nicht neu, sie hat aber durch das Urteil des VG Gießen vom 22. Januar 2020 - 1 K 6019/18. GI - eine gewisse Zuspitzung erfahren. ${ }^{4}$ Das Gericht hat mit seinem Urteil, gegen das mittlerweile Berufung eingelegt worden ist, die Genehmigung von drei Windenergieanlagen mit einer Nabenhöhe von 149 Metern, einem Rotordurchmesser von 115 Metern und einer Nennleistung von 3,0 Megawatt wegen der Verletzung der europäischen Vogelschutzrichtlinie 2009/147/EG aufgehoben. ${ }^{5}$ Das Gericht war der Auffassung, der Betrieb der Anlagen stelle eine Verletzung des Tötungsverbots aus $₫ 44$ Abs. 1 Nr. 1 Bundesnaturschutzgesetz (BNatSchG) dar, die auch nicht

Prof. Dr. Ekkehard Hofmann,

Inhaber des Lehrstuhls für Öffentliches Recht,

insbesondere Umweltrecht,

und Leiter des dortigen Forschungsschwerpunkts Recht

des Klimawandels,

Universität Trier, Trier, Deutschland im Wege einer Ausnahme in europarechtskonformer Weise genehmigt werden könne. Sollte sich diese Rechtsauffassung durchsetzen, würde das schon wegen des umfassenden Schutzansatzes der Vogelschutzrichtlinie eine erhebliche weitere Hürde beim ohnehin ins Stocken geratenen Ausbau der Windenergie als Element des Klimaschutzkonzeptes der Bundesregierung bedeuten. ${ }^{6}$ Daher ist im Folgenden zu untersuchen, ob und inwiefern Möglichkeiten bestehen, den umschriebenen und nicht stets vermeidbaren Zielkonflikt ${ }^{7}$ unter Beachtung der Vorgaben des europäischen Naturschutzrechts so zu lösen, dass der Ausbau der erneuerbaren Energien - namentlich der der Windenergie an Land - von seinem Umfang her kompatibel mit den Zielen des Klimaschutzes erfolgen kann.

^) Der Beitrag beruht auf einem Rechtsgutachten des Verfassers im Auftrag des Kompetenzzentrums Naturschutz und Energiewende (KNE). Ich danke meinem Team, allen voran Benjamin Gwiasda und Mariana Schikova, ganz herzlich für die zuverlässige und engagierte Unterstützung

1) Siehe Art. 2 (1) lit. (a) des Übereinkommens von Paris, ABl. 2016 L 282, S. 4, 5. Wie hochbrisant die Lage schon jetzt ist, belegen Studien, die für das Jahr 2025 einen $\mathrm{CO}_{2}-$ Gehalt in der Luft vorhersagen, wie er auf der Erde das letzte Mal vor 3,3 Millionen Jahren beobachtet werden konnte, s. de la Vega/Chalk/Wilson et al., 10 Sci Rep 2020, 11002, Stand 10.10.2020, abrufbar unter https:// doi.org/10.1038/s41598-020-67154-8.

2) Siehe jüngst den Bericht der European Environment Agency, State of nature in the EU, Results from reporting under the nature directives 2013-2018, EEA-Report 10/2020, 2020, Stand 21.10.2020, abrufbar unter https://www.eea.europa.eu/publications/state-ofnature-in-the-eu-2020

3) $\mathrm{Zu}$ optimistisch allerdings die Beurteilung der Spannungslage durch die Europäische Kommission, Commission Staff Working Document, Fitness Check of the EU Nature Legislation (Birds and Habitats Directives), Directive 2009/147/EC of the European Parliament and of the Council of 30.11.2009 on the conservation of natural habitats and of wild fauna and flora, SWD(2016) 472 final, S. 6 f.

4) $\mathrm{NuR} 2020,206-214$

5) Richtlinie 2009/147/EG des Europäischen Parlaments und des Rates vom 30.11.2009 über die Erhaltung der wildlebenden Vogelarten, AB1. 2010 L 20, S. 7.

6) Quantifizierte Ausbauziele für die Windenergie in Bundesregierung, Klimaschutzprogramm 2030 der Bundesregierung zur Umsetzung des Klimaschutzplans 2050, 2019, Stand 10.10.2020, abrufbar unter https://www.bundesregierung.de/resource/blob/ 975226/1679914/e01d6bd855f09bf05cf7498e06d0a3ff./2019-1009-klima-massnahmen-data.pdf?download=1, S. 39.

7) Anders offenbar die Auffassung der Europäischen Kommission, die auf dem Gebiet des Naturschutzrechts und des Klimaschutzes ein kohärentes System zu erkennen vermag, das allenfalls unter Bedrohungen seitens (land-)wirtschaftlicher Interessen leidet, Europäische Kommission, Commission Staff Working Document, Fitness Check of the EU Nature Legislation (Birds and Habitats Directives), Directive 2009/147/EC of the European Parliament and of the Council of 30.11 .2009 on the conservation of wild birds and Council Directive $92 / 43 /$ EEC of 21.5.1992 on the conservation of natural habitats and of wild fauna and flora, SWD(2016) 472 final, S. $6 \mathrm{f}$. 


\section{Die artenschutzrechtliche Zulässigkeit von Windenergieanlagen an Land}

Die Überlegungen des VG Gießen gehen davon aus, dass die signifikante Erhöhung des Tötungsrisikos für geschützte Vogelarten das Tötungsverbot verletzt. Da die Vogelschutzrichtlinie ausweislich ihres Art. 5 (nur) die absichtliche Tötung verbietet, versteht sich das nicht von selbst.

\subsection{Das Tötungsverbot der Vogelschutzrichtlinie}

\subsubsection{Absicht als Merkmal des subjektiven Tatbestands}

Art. 5 der Vogelschutzrichtlinie verpflichtet die Mitgliedstaaten, die erforderlichen Maßnahmen zur Schaffung einer allgemeinen Regelung zum Schutz aller in Europa wildlebenden Vogelarten nach Artikel $1 \mathrm{zu}$ erlassen, insbesondere Verbote des absichtlichen Tötens oder Fangens, ungeachtet der angewandten Methode. Unter diesen Tatbestand des absichtlichen Tötens fällt eindeutig die auf die Tötung gerichtete Jagd, die auch im Zentrum der Entstehung des Berner Übereinkommens ${ }^{8}$ und der das Berner Übereinkommen im Europarecht umsetzende Vogelschutzrichtlinie gestanden haben dürfte. ${ }^{9} \mathrm{Da}$ Windenergieanlagen keine Einrichtungen darstellen, die zur Jagd von Vögeln dienen oder die ihre Tötung bezwecken, ist vorgeschlagen worden, die durch Kollision gelegentlich des Betriebs einer Windenergieanlage getöteten Vögel nicht als Resultat einer nach Art. 5 lit. a) V-RL verbotenen Tötungshandlung anzusehen. ${ }^{10}$ Damit wären Windenergieanlagen artenschutzrechtlich von der Rechtfertigung für eine Tötungshandlung im Sinne der Vogelschutzrichtlinie befreit.

Das hat sich jedoch in der Rechtsprechung des EuGH (bislang) nicht durchsetzen können, wonach jedenfalls im Kontext der FFH-Richtlinie der Tatbestand des absichtlichen Tötens bereits im Falle einer billigen Inkaufnahme erfüllt ist. ${ }^{11}$ Eine Übertragung auf die Vogelschutzrichtlinie entspricht wohl der nachstehend erläuterten herrschenden Ansicht in Deutschland, ist aber nicht unproblematisch, da sich die Rechtfertigungsgründe von FFH- und Vogelschutzrichtlinie erheblich unterscheiden und sich daraus ein unterschiedliches Schutzniveau ergibt, das wegen seiner Wertungswidersprüche befremdet. ${ }^{12}$

Der ursprünglich von der Rechtsprechung entwickelte Signifikanz-Ansatz ${ }^{13}$ hat mit der Novelle des BNatSchG im Jahre 2017 auch Eingang in die gesetzliche Regelung des $\$ 44$ Abs. 5 Nr. 1 BNatSchG gefunden. Eine Differenzierung des Tatbestands jeweils nach FFH- oder Vogelschutzrichtlinie erfolgt dabei nicht. Erforderlich ist damit auf objektiver Seite ein ursächlicher Zusammenhang zwischen dem Betrieb der Anlage und einer hinreichend erhöhten Tötungswahrscheinlichkeit, die anhand der artspezifischen Verhaltensweisen, der Häufigkeit des Aufenthaltes im Gefährdungsbereich und der Wirksamkeit vorgesehener Schutzmaßnahmen zu bewerten ist. ${ }^{14}$ Für den subjektiven Tatbestand genügt ein billigendes Inkaufnehmen eines Kollateralschadens.

Die Erfüllung des so verstandenen objektiven Tatbestands ist im Falle von Windenergieanlagen nicht kategorisch auszuschließen, das Vorliegen eines Eventualvorsatzes dann stets anzunehmen, wenn die signifikante Erhöhung der Tötungswahrscheinlichkeit ornithologisch zu bejahen ist und die Anlage trotzdem errichtet werden soll. Dem folgt auch das VG Gießen. ${ }^{15}$ Es sprechen aber nachvollziehbare Überlegungen dafür, im Anwendungsbereich der Vogelschutzrichtlinie dem Erhaltungszustand mit Blick auf den Absichtsbegriff größere Beachtung zu schenken (s. sogleich 2.1.2).

\subsubsection{Berücksichtigung des Erhaltungszustands} betroffener Populationen

Angesichts der unterschiedlichen Konzeption von FFHund Vogelschutzrichtlinie verdient der jüngst unterbreitete
Vorschlag der Generalanwältin Kokott besondere Beachtung, den Absichtsbegriff abhängig vom Erhaltungszustand der betroffenen Arten zu differenzieren. ${ }^{16}$ Ihr Vorschlag geht dahin, in den Fällen, in denen eine Tötung nicht bezweckt, sondern nur in Kauf genommen wird, die Verbote nach Art. 5 lit. a) und b) der Vogelschutzrichtlinie nur gelten zu lassen, soweit dies notwendig ist, um diese Arten im Sinne von Art. 2 auf einem Stand zu halten oder auf einen Stand zu bringen, der insbesondere den ökologischen, wissenschaftlichen und kulturellen Erfordernissen entspricht, und dabei den wirtschaftlichen und freizeitbedingten Erfordernissen Rechnung trägt. ${ }^{17}$

Für diese Auffassung spricht sicherlich, dass die FFH-Richtlinie anders als die Vogelschutzrichtlinie mit den ,zwingenden Gründen des überwiegenden öffentlichen Interessen einschließlich solcher sozialer oder wirtschaftlicher Art" einen sehr weiten „Auffangtatbestand“ zur Erteilung von Ausnahmen im Falle von unvermeidbaren Tötungen zugunsten gewichtiger Gründe enthält. Ein konsistenter Sprachgebrauch wäre es aber prima facie nicht, den Begriff der Absicht in beiden Richtlinien so unterschiedlich $\mathrm{zu}$ interpretieren. Auch erscheint noch nicht restlos geklärt, wie sich diese Neuinterpretation des Absichtsbegriffs unter Einbeziehung der Ziele der Vogelschutzrichtlinie nach Art. 2 V-RL zum Verschlechterungsverbot des Art. $13 \mathrm{~V}$-RL verhielte, das die inkriminierten Handlungen weitestgehend ohnehin verbieten dürfte. Kokotts Vorschlag läuft im Ergebnis darauf hinaus, dass eine Inkaufnahme der Erhöhung des Tötungsrisikos keine Tötung darstellen soll, wenn und soweit sich ein guter Erhaltungszustand im Sinne des Art. 2 V-RL infolge des erhöhten Tötungsrisikos nicht verschlechtert, oder aber die Erreichung eines mit Art. 2 V-RL kompatiblen Zustands nicht erschwert würde. Hinsichtlich der ersten Variante würde das zur Funktionslosigkeit von Art. 13 V-RL führen. Es bleibt deshalb abzuwarten, ob sich der EuGH dieser Auffassung anschließen wird. Sollte er es nicht tun, bliebe es dabei, dass bereits die beiläufige Tötung geschützter Vogelarten grundsätzlich rechtfertigungsbedürftig ist.

Die Ausnahmetatbestände des Art. 9 Abs. 1 V-RL stehen daher im Zentrum der nachstehenden Betrachtung.

8) Übereinkommen über die Erhaltung der europäischen wildlebenden Pflanzen und Tiere und ihrer natürlichen Lebensräume (Berner Übereinkommen) v. 19.9.1979, BGBl. 1984 II S. 618, 620

9) Vgl. Art. 9 des Berner Übereinkommens und Art. 9 der Richtlinie 2009/147/EG des Europäischen Parlaments und des Rates vom 30.11.2009 über die Erhaltung der wildlebenden Vogelarten (Vogelschutzrichtlinie), ABl. 2010 L 20, S. 7, $10 \mathrm{f}$

10) In diesem Sinne wohl Sobotta, NuR 2007, 642, $648 \mathrm{f}$

11) EuGH, Urt. v. 30.1.2002 - C-103/00, Slg. 2002, I-1147, Rdnr. 36 - Kommission/Griechenland (Caretta caretta); EuGH, Urt. v. 18.5.2006 - C-221/04, Slg. 2006, I-4515, Rdnr. 71 Kommission/Spanien (Schlingenjagd).

12) Für Sobotta ist die Übertragung der Maßstäbe der FFH-Richtlinie auf die Vogelschutzrichtlinie ein ,provozierendes Ergebnis“, Sobotta, NuR 2007, 642, 648 r. Sp.; s.a. auch die Warnung von Generalanwältin Kokott vor einer Übertragung des Absichtsbegriffs von der FFH-Richtlinie auf die Vogelschutzrichtlinie, Schlussanträge v. 19.9.2020 - C-473/19 u.a., ECLI:EU:C:2020:699, Rdnr. 39

13) BVerwG, Urt. v. 9.7.2008 - 9 A 14/07, juris Rdnr. 91; Urt v. 28.4.2016 - 9 A 9/15, juris Rdnr. 141; Urt. v. 10.11.2016 9 A 18/15, juris Rdnr. 82f.; Urt. v. 6.4.2017 - 4 A 16/16, juris Rdnr. 73; aus jüngerer Zeit BVerwG, Beschl. v. 8.3.2018 - 9 B 25/17, juris Rdnr. 11 (st. Rspr.).

14) BVerwG, Urt. v. 14.7.2011 - 9 A 12/10, juris Rdnr. 99; Urt. v. 18.3.2009 - 9 A 39/07, juris Rdnr. 58.

15) VG Gießen, Urt. v. 22.1.2020 - 1 K 6019/18.GI, juris Rdnr. 87.

16) Generalanwältin Kokott, Schlussanträge v. 10.9.2020 - C-473/19 u. a., ECLI:EU:C:2020:699, Rdnr. 93.

17) Generalanwältin Kokott, Schlussanträge v. 10.9.2020 - C-473/19 u. a., ECLI:EU:C:2020:699, Rdnr. 93. 


\subsection{Die Ausnahmetatbestände des Art. 9 Abs. 1 V-RL}

2.2.1 Die Korrektur der Ausnahmetatbestände der Vogelschutzrichtlinie mit Hilfe ungeschriebener Ausnahmegründe?

\subsubsection{Die unterschiedlichen Schutzkonzepte}

Die Reichweite der Rechtfertigungsgründe des Art. 9 Abs. 1 V-RL hängt fundamental von der Einbettung in das Gesamtkonzept der Richtlinie ab, das sich von dem der FFH-Richtlinie deutlich unterscheidet. Während die Vogelschutzrichtlinie alle europäischen Vogelarten ihrem differenzierten Schutz unterstellt und dabei der praktischen Rechtsanwendung zur Lösung von Konfliktfällen ein strenges Konzept der Ausnahmeerteilung bereitstellt, unterscheidet die FFH-Richtlinie zwischen verschiedenen Tierarten nach Schutzbedürftigkeit und erlaubt ihre Beeinträchtigung unter bestimmten, im Einzelnen aufgezählten Umständen, aber eben auch im Falle des Vorliegens zwingender Gründe des überwiegenden öffentlichen Interesses auch sozialer und wirtschaftlicher Art. ${ }^{18}$

\subsubsection{Harmonisierung der Ausnahmetatbestände in Art. 9 Abs. 1 V-RL mit Hilfe des Primärrechts?}

Dies führt angesichts der Bevorzugung des Fangs von Vögeln zu Liebhaberzwecken gegenüber der nur mit der Argumentation des VG Gießen schwer zu begründenden oder gar nicht zulässigen Verwirklichung von Infrastrukturprojekten im öffentlichen Interesse zu Ergebnissen, die als Wertungswiderspruch empfunden werden. Während das VG Gießen davon ausgeht, dass der europäische Gesetzgeber die unterschiedliche Konzeptionierung bewusst vorgenommen habe und diese daher hinzunehmen sei, ${ }^{19}$ haben andere Stimmen die beobachteten Schutzkonzeptdifferenzen zum Anlass für Überlegungen genommen, auf welchem Weg eine Harmonisierung erreicht werden könnte. ${ }^{20}$ So wird vorgeschlagen, die ,zwingenden Gründe des überwiegenden öffentlichen Interesses" aus der (jüngeren) FFH-Richtlinie auf die Vogelschutzrichtlinie zu übertragen, um insbesondere zu vermeiden, dass im Ergebnis das Schutzniveau in einem faktischen Vogelschutzgebiet infolge von Art. 7 und 16 FFH-Richtlinie höher liegt als einem förmlich festgesetzten, für das die Ausnahmevorschriften der FFH-Richtlinie gelten. ${ }^{21}$ Das darf als systematische Auslegung von Art. 9 Abs. 1 V-RL ,im Lichte von" Art. 16 FFH-RL bewertet werden, die sicher einiges für sich hat, der der EuGH aber bislang nicht beigetreten ist.

Verschiedentlich wird ein Ausweg über die Annahme eines ungeschriebenen Rechtfertigungsgrundes in Art. 9 Abs. 1 V-RL gesehen. Hierfür wird mit dem Verhältnismäßigkeitsgrundsatz und der willkürlichen Ungleichbehandlung verschiedener Tierarten argumentiert, ${ }^{22}$ zum Teil wird unmittelbar auf die skizzierten Wertungswidersprüche zwischen beiden Richtlinien hingewiesen. ${ }^{23}$ Gleichgültig jedoch, auf welchem konstruktiven Weg eine Harmonisierung erreicht werden soll, das Ergebnis einer interpretatorischen Ergänzung der Vogelschutzrichtlinie ist mit der Rechtsprechung des EuGH nicht in Einklang zu bringen, der in dem Katalog des Art. 9 Abs. 1 V-RL eine abgeschlossene Enumeration möglicher Ausnahmen erkennt. ${ }^{24}$ Mit der Annahme eines solchen abgeschlossenen Katalogs wäre die Erweiterung um ungeschriebene Rechtfertigungsgründe von vornherein nicht vereinbar. Im Übrigen steht dieser Befund auch dem eben erläuterten Vorschlag Berkemanns entgegen, die Ausnahmetatbestände der FFH-Richtlinie auf die Vogelschutzrichtlinie zu übertragen. ${ }^{25}$ Auch das wäre eine Erweiterung der Rechtfertigungsmöglichkeiten gegenüber dem mit der Rechtsprechung des EuGH abgeschlossenen Katalogs an Tatbeständen in Art. 9 Abs. 1 V-RL.

\subsubsection{Würdigung anhand der Rechtsprechung des EuGH}

Der EuGH verdient in seiner Rechtsprechung zur Vogelschutzrichtlinie auch Zustimmung. Der Katalog an Gründen, die eine Ausnahme tragen können, muss einer verlässlichen Beschränkung unterworfen sein. Dass Art. 9 Abs. 1
V-RL so gemeint ist, belegt auch der Wortlaut der Bestimmung, nach der die Aufzählung der Ausnahmetatbestände keinen Hinweis enthält, es würde nur eine Auswahl besonders wichtiger Ausnahmegründe aufgezählt (etwa durch „insbesondere“ oder ähnliche Formulierungen).

Der EuGH hat sich an unterschiedlichen Ansätzen in der Vogelschutzrichtlinie einerseits und der FFH-Richtlinie andererseits bislang nicht gestört, so dass die Annahme eines Verstoßes gegen das primärrechtliche Gleichbehandlungsgebot aus Art. 20 EU-GRCh oder das Kohärenzgebot des Art. 7 AEUV keinen Anhalt in der Judikatur des EuGH fände, zumal der hier angesprochene Gesetzgeber sicher über Gestaltungsspielräume verfügt, deren Verletzung erst dann in der Justiz moniert wird, wenn sich gar kein vernünftiger Grund für die Differenzierung finden lässt. ${ }^{26}$ Das wäre hier nicht zu erwarten.

Gleiches gälte für die Bemühung des Verhältnismäßigkeitsgrundsatzes nach Art. 5 Abs. 4 EUV. ${ }^{27}$ Die UMK argumen-

18) Übersichtliche Zusammenstellung der Unterschiede bei Sailer, Gesetzgeberische Handlungsmöglichkeiten zur Weiterentwicklung der artenschutzrechtlichen Ausnahmeregelung, Hintergrundpapier, Würzburger Berichte zum Umweltenergierecht, Nr. 49 v. 11.3.2020, Stand 10.10.2020, abrufbar unter https:// stiftung-umweltenergierecht.de/wp-content/uploads/2020/03/ Stiftung_Umweltenergierecht_WueBerichte_49_Artenschutzrechtliche_Ausnahme_2020-03-11.pdf, S. 4.

19) VG Gießen, Urt. v. 22.1.2020 - 1 K 6019/18.GI, juris Rdnr. 109

20) Müller/Klostermeier, NVwZ 2020, 774, $775 \mathrm{f}$; ebenso bereits Gellermann, in: Landmann/Rohmer, Umweltrecht, Kommentar, 92. EL, Stand Februar 2020, BNatSchG, \$45 Rdnr. 24; ders., NuR 2020, 178, 181; ders., in: Schrödter, Baugesetzbuch, Kommentar, 9. Aufl. 2019, S1a Rdnr. 219 m. w. N.; Berkemann, in: ders./Halama, Handbuch zum Recht der Bau- und Umweltrichtlinien der EU, 2. Aufl 2011, S. 816f. Rdnr. 253 (das strenge Schutzregime der Vogelschutzrichtlinie entbehre der sachlichen Rechtfertigung).

21) Berkemann, in: ders./Halama, Handbuch zum Recht der Bauund Umweltrichtlinien der EU, 2. Aufl. 2011, S. 816f. Rdnr. 253

22) Lau, NuR 2013, 685, 688; Philipp, NVwZ 2008, 593, 597; Karpenstein/Engel, ZUR 2020, 437, 438; ähnlich wohl auch VGH München, Urt. v. 19.2.2014-8 A 11.40040, juris Rdnr. 846-849.

23) Müller/Klostermeier, NVwZ 2020, 774, 775.

24) Zuletzt sehr deutlich EuGH, Urt. v. 26.1.2012 - C-192/11, ECLI:EU:C:2012:44, Rdnr. 39 („,..énumérés de manière exhaustive à l'article 9...") - Kommission/Polen; zuvor schon EuGH, Urt. v. 8.7.1987 - C-247/85, Slg. 1987, 3029, Rdnr. 7, 58 - Kommission/Belgien; EuGH, Urt. v. 7.3.1996 - C-118/94, Slg. 1996, I-1223, Rdnr. 21 - Associazione Italiana per il WWF; Schütte/Gerbig, in: Schlacke, Gemeinschaftskommentar zum Bundesnaturschutzgesetz, 2. Aufl. 2017, \45 Rdnr. 32

25) Berkemann, in: ders./Halama, Handbuch zum Recht der Bauund Umweltrichtlinien der EU, 2. Aufl. 2011, S. 816f. Rdnr. 253.

26) Vgl. zu Art. 20 EU-GRCh etwa EuGH, Urt. v. 13.11.1990 C-370/88, Slg. 1990, I-4071, Rdnr. 24; EuGH, Urt. v. 13.12. 1984 - C-129/82 u.a., Slg. 1984, 4127, Rdnr. 19ff.; EuGH, Urt. v. 6.7.1983 - C-117/81, Slg. 1983, 2191, Rdnr. 14; EuGH, Urt. v. 19.10.1977 - C-117/76 u.a., Slg. 1977, 1753, Rdnr. 7f.; zur eingeschränkten Justiziabilität bzgl. Art. 7 AEUV vgl. Obwexer, in: Groeben/Schwarze/Hatje, Europäisches Unionsrecht, Vertrag über die Europäische Union, Vertrag über die Arbeitsweise der Europäischen Union, Charta der Grundrechte der Europäischen Union, 7. Aufl. 2015, AEUV, Art. 7 Rdnr. 15 sowie Ruffert, in: Calliess/Ruffert, EUV, AEUV, das Verfassungsrecht der Europäischen Union mit Europäischer Grundrechtecharta, Kommentar, 5. Aufl. 2016, AEUV, Art. 7 Rdnr. 5 sowie Streinz, in: Streinz, EUV/AEUV, Vertrag über die Europäische Union, Vertrag über die Arbeitsweise der Europäischen Union, Charta der Grundrechte der Europäischen Union, 3. Aufl. 2018, AEUV, Art. 7 Rdnr. 8 sowie Schorkopf, in: Grabitz/Hilf/Nettesheim, Das Recht der Europäischen Union, 70. EL, Stand Mai 2020, AEUV, Art. 7 Rdnr. 14

27) Umweltministerkonferenz, 94. UMK-Bericht, Bericht zu TOP 4 Ziffer 4, Hinweise zu den rechtlichen und fachlichen Ausnahmevoraussetzungen nach $₫ 45$ Abs. 7 BNatSchG bei der Zulassung von Windenergievorhaben, 2020, Stand 10.10.2020, abrufbar unter https://www.umweltministerkonferenz.de/documents/ anlage-zu-top-4,-ziff-4_1591168257.pdf, S. 3. 
tiert, eine Gleichstellung der Ausnahmemöglichkeiten von Vogelschutzrichtlinie und FFH-Richtlinie sei erforderlich. ${ }^{28}$ Bislang hat der EuGH hingegen, soweit ersichtlich, noch nicht einmal angedeutet, dass er die Ausnahmetatbestände der Vogelschutzrichtlinie mit Blick auf den Verhältnismäßigkeitsgrundsatz oder aus anderen Gründen als unzureichend bewertet. Eine Harmonisierung im Wege des Hinzufügens eines ungeschriebenen Ausnahmegrundes für den Ausbau der Windenergie ist ohnehin nur dann erforderlich, wenn sich tatsächlich kein passender Rechtfertigungsgrund in der Vogelschutzrichtlinie finden lässt. Daher ist die Analyse vor allem auf den Wortsinn des Art. 9 Abs. 1 V-RL zu richten.

\subsubsection{Die Ausnahmetatbestände im Einzelnen}

Die Ausnahmetatbestände sind mit vorstehenden Überlegungen in Art. 9 Abs. $1 \mathrm{~V}-\mathrm{RL}$ abschließend aufgeführt. Auch Art. 2 V-RL enthält keine über Art. 9 V-RL hinausgehenden Rechtfertigungsgründe, ${ }^{29}$ gebietet jedoch zu berücksichtigen, dass die Vogelschutzrichtlinie im Spannungsfeld zwischen den Interessen des Naturschutzes und anderen, zum Teil gegenläufigen Interessen kultureller, wirtschaftlicher und freizeitbezogener Art steht, denen ebenfalls Rechnung getragen werden muss. Im Einzelnen ist Folgendes zu bemerken:

\subsubsection{Gesundheit und öffentliche Sicherheit (1. Spiegelstrich)}

Art. 9 Abs. 1 lit. a) 1. Spiegelstrich V-RL bestimmt, dass die Mitgliedstaaten, sofern es keine andere zufriedenstellende Lösung gibt, von den Artikeln 5 bis 8 im Interesse der Gesundheit und der öffentlichen Sicherheit abweichen können.

\subsection{Gesundheit}

\section{Begriff}

Mit dem Begriff der Gesundheit dürfte sich die Richtlinie auf die menschliche Gesundheit beziehen, ${ }^{30}$ auf die es schon lange gut belegte Auswirkungen des Klimawandels gibt. Trotz der mittel- und langfristigen Entwicklung im Bereich der globalen Erwärmung sind die naturwissenschaftlich hoch anspruchsvollen ursächlichen Zusammenhänge und die zu erwartenden Folgen im Grundsatz bekannt. Schon im Jahre 2009 urteilte das Umweltbundesamt, die Auswirkungen des Klimawandels hätten „,bereits heute" vielfältige direkte und indirekte Effekte auf die menschliche Gesundheit. ${ }^{31}$ Damit bezog sich das Umweltbundesamt auf das erhöhte Auftreten wärmeliebender und zum Teil schädlicher Organismen wie etwa Ambrosiapollen und Brennhaare von Nachtfaltern. Gesundheitsrisiken liegen aber auch in erhöhten Mortalitätsraten infolge von klimabedingten Hitzewellen, die empfindliche Bevölkerungsteile besonders hart treffen, sowie gesundheitsbezogene Gefahren aus Hochwasserereignissen. Diese Auswirkungen lassen sich in den Prognosen mittlerweile regional spezifizieren. ${ }^{32}$

Die Bundesregierung hatte sich jedenfalls schon im Jahr 2013 veranlasst gesehen, das Umweltbundesamt (UBA) und das Robert-Koch-Institut (RKI) um die Erarbeitung eines Ratgebers zu ,Klimawandel und Gesundheit“ als allgemeinen Rahmen für Handlungsempfehlungen für Behörden und weitere Akteure zu bitten. ${ }^{33}$ In ganz ähnlicher Herangehensweise identifiziert und quantifiziert ein von der Europäischen Kommission im Jahre 2014 veröffentlichter Bericht verschiedene Gesundheitsrisiken infolge des Klimawandels. ${ }^{34}$ Auch die Weltgesundheitsorganisation sieht diese klimawandelbedingten Gefahren für die Gesundheit der Bevölkerung. ${ }^{35}$ Eine Maßnahme, die wie die Errichtung einer Windenergieanlage einen - wenn auch einzeln betrachtet kleinen - Beitrag zum Klimaschutz leistet, liegt damit wegen ihrer Zielrichtung eindeutig im Interesse des so verstandenen Gesundheitsschutzes.
Es gibt keinen Anlass, dies für den Kontext der Vogelschutzrichtlinie anders zu beurteilen. Jedenfalls gibt die Entstehungsgeschichte der Vogelschutzrichtlinie weder in ihrer ursprünglichen Gestalt noch in ihrer Novelle aus dem Jahre 2009 Anhaltspunkte dafür her, dass dem Gesetzgeber der Zusammenhang von Artenschutz, Klimawandel und Gesundheit bewusst gewesen sein könnte. Sie erzeugt in der Rechtsprechung des EuGH ohnehin keine Bindung im Sinne einer subjektiv-historischen Auslegung. ${ }^{36}$ Darüber hinaus dient die Heranziehung semantisch vager Begriffe auch dazu, abstrakte Regelungen zukunftsoffen auszugestalten, so dass sie es erlauben, neue Entwicklungen einer problemadäquaten Lösung zuzuführen. Dazu dürfte die dramatische Verschärfung der Klimaproblematik insbesondere im vergangenen Jahrzehnt ohne weiteres zählen, zumal der Ausbau der Erneuerbaren Energien in jüngerer Zeit vom EuGH als ,„übergeordnetes öffentliches Interesse“ anerkannt worden ist. ${ }^{37}$

Des Weiteren ist das Klimaschutzrecht mittlerweile im Primärrecht der EU höchstrangig und so persistent verankert, dass eine Auslegung des Gesundheitsbegriffs im Rahmen von Art. 9 Abs. 1 V-RL, die die entsprechenden Auswirkungen des Klimawandels ignoriert, kaum mit den Zielen des Primärrechts in Einklang zu bringen wäre. Für die Berücksichtigung der gesundheitlichen Konsequenzen des Klimawandels spricht schon, dass Art. 191 Abs. 1 AEUV in Bezug auf die Umweltpolitik der Union die Ziele des Gesundheitsschutzes (Art. 191 Abs. 1 2. Spiegelstrich) und die der Bekämpfung des Klimawandels (Art. 191 Abs. 1

28) Umweltministerkonferenz, 94. UMK-Bericht, Bericht zu TOP 4 Ziffer 4, Hinweise zu den rechtlichen und fachlichen Ausnahmevoraussetzungen nach $\$ 45$ Abs. 7 BNatSchG bei der Zulassung von Windenergievorhaben, 2020, Stand 10.10.2020, abrufbar unter https://www.umweltministerkonferenz.de/documents/anlage-zu-top-4,-ziff-4_1591168257.pdf, S. 3.

29) EuGH, Urt. v. 8. 7.1987 - C-262/85, Slg. 1987, 3073, Rdnr. 8 Kommission/Italien; wohl zustimmend Sobotta, NuR 2007, 642, 649.

30) So jedenfalls die deutsche Umsetzung in $₫ 45$ Abs. 7 S. 1 Nr. 4 BNatSchG.

31) Umweltbundesamt, Klimawandel und Gesundheit, Welche Probleme verursachen Wärme liebende Schadorganismen?, Abschlussbericht, Internationales UBA/BMU-Fachgespräch: 9 und 10.11.2009, UBA, Berlin-Dahlem, 2010, Stand 11.10.2020, abrufbar unter https://www.umweltbundesamt.de/sites/default/ files/medien/publikation/long/3925.pdf, S. 11.

32) Deutscher Wetterdienst/Extremwetterkongress Hamburg, Was wir heute über das Extremwetter in Deutschland wissen, Stand der Wissenschaft zu extremen Wetterphänomenen im Klimawandel in Deutschland, 2020, Stand 11.10.2020, abrufbar unter https:// www.dwd.de/DE/presse/ewk_hamburg/downloads/ewk_papier.pdf?_blob=publicationFile $\& v=2$, S. 7.

33) Umweltbundesamt/Robert Koch-Institut, Klimawandel und Gesundheit, Allgemeiner Rahmen zu Handlungsempfehlungen für Behörden und weitere Akteure in Deutschland, 2013, Stand 11.10.2020, abrufbar unter https://www.rki.de/DE/Content/ Kommissionen/UmweltKommission/Stellungnahmen_Berichte/Downloads/klimawandel_gesundheit_handlungsempfehlungen_2013.pdf?__blob=publicationFile.

34) Paci, Human Health Impacts of Climate Change in Europe, Report for the PESETA II project, 2014, Stand 11.10.2020, abrufbar unter https://publications.jrc.ec.europa.eu/repository/bitstream/JRC86970/1fna26494enn.pdf, passim, insbes. S. $20 \mathrm{f}$

35) World Health Organization Regional Office for Europe, Protecting Health in Europe from Climate Change: 2017 Update, 2017, Stand 11.10.2020, abrufbar unter https://www.euro.who.int/_ data/assets/pdf_file/0004/355792/ProtectingHealthEuropeFromClimateChange.pdf, S. $2 \mathrm{ff}$.

36) Instruktiver, wenn auch nicht mehr ganz aktueller Überblick bei Seyr, Der effet utile in der Rechtsprechung des EuGH, 2008, S. $94 \mathrm{ff}$.

37) EuGH, Urt. v. 4.5.2016 - C-346/14, ECLI:EU:C:2016:322, Rdnr. 71 - Kommission/Österreich (im Kontext der WRRL und eines Wasserkraftwerks). 
4. Spiegelstrich am Ende) gleichberechtigt nebeneinanderstellt. Was die für den Ausbau der Windenergie einschlägige Förderung der Erneuerbaren Energien angeht, so ist sie ebenfalls auf höchster Ebene zu beachten (Art. 194 Abs. 1 lit. c AEUV). Begrifflich liegt damit bei dem Klimaschutz dienenden Windenergieanlagen ein hinreichender Bezug zur Gesundheit im Sinne von Art. 9 Abs. 1 V-RL vor.

„Im Interesse von“: Kausalität

Notwendig ist aber auch, dass die Maßnahme, zu deren Gunsten eine Ausnahme von den Tötungs- und Beeinträchtigungsverboten gemacht werden soll, ,,im Interesse“ der Gesundheit erfolgt. Die Formulierung verweist auf eine Zwecksetzung der jeweiligen Maßnahme, die auf die entsprechende Absicht zielt. Die reine Behauptung einer richtlinienkonformen Zwecksetzung dürfte aber nicht genügen; es muss auch objektiv plausibel sein, dass die fragliche Handlung die beabsichtigten Wirkungen haben kann. Mit dieser Überlegung verlangen die Tatbestände des Art. 9 Abs. $1 \mathrm{~V}-\mathrm{RL}$ zu ihrer Verwirklichung, dass die fragliche Maßnahme einen ursächlichen Beitrag zur Förderung des in Rede stehenden Rechtsguts leistet. Hier setzt Gellermann an, wenn er argumentiert, der Bau der einzelnen Windenergieanlage habe keinen nachweisbaren Effekt im Sinne eines Beitrags zum Klimaschutz. ${ }^{38}$ Die Folgen des Klimawandels ließen noch Jahrzehnte auf sich warten und der ursächliche Zusammenhang zwischen der Errichtung einer Windenergieanlage und der Abwendung der globalen Erwärmung sei höchstens minimal. ${ }^{39}$ Eine solche Entscheidung unter Unsicherheit könne nicht zur Rechtfertigung einer greifbaren Erhöhung des Tötungsrisikos für europäische Vogelarten herangezogen werden, das sich nicht in diesem Sinne ,verrechnen“ ließe. ${ }^{40}$ Plakativ formuliert Gellermann, es sei im Rahmen von $\ 45$ Abs. 7 S. 1 Nr. 4 BNatSchG kein Raum für umweltpolitisch fragwürdige Verrechnungen, die effektive Verluste im Bereich der Artenvielfalt durch potenzielle Vorteile für das Klima aufzuwiegen versuchten. ${ }^{41}$

In eine ähnliche Richtung zielen weitere Stimmen in der Literatur, nach der Rechtsprechung des EuGH ${ }^{42}$ seien konkrete positive Auswirkungen auf die Umwelt gefordert, was bei den mittelbaren, kausal nicht nachweisbaren Effekten des Betriebs einer Windenergieanlage zu verneinen sei. ${ }^{43} \mathrm{Ob}$ das als Auslegung des in dem genannten Urteil noch nicht einmal erwähnten Art. 9 Abs. 1 V-RL betrachtet werden kann, müsste für die Übertragung auf die vorliegende Problematik genauso hergeleitet werden, wie der Transfer der Begründung des EuGH zur Anerkennung der Verkleinerung eines Vogelschutzgebiets in der Leybucht-Entscheidung wegen positiver Folgen für die Umwelt auf den Begriff der Gesundheit in Art. 9 Abs. 1 lit. a) 1. Spiegelstrich V-RL. Das wäre nicht unproblematisch. Gatz verkennt jedenfalls, dass die konkreten positiven Auswirkungen der Schutzgebietsverkleinerung auf die Vogelwelt in der Leybucht-Entscheidung eine hinreichende Bedingung für deren Zulässigkeit darstellen, nicht eine notwendige. ${ }^{44}$ Aber selbst unter der Annahme, dass beides im Ergebnis trägt, folgt daraus nicht, dass eine Windenergieanlage, die zusammen mit anderen Windenergieanlagen hilft, ein mitgliedstaatliches Programm zur Erfüllung der Verpflichtungen aus dem Pariser Übereinkommen verwirklichen, wegen ihres einzeln betrachtet nur kleinen Beitrags zum Klimaschutz nicht gerechtfertigt werden könnte.

Dem Argument der fehlenden Kausalität muss nämlich entschieden entgegengehalten werden, dass in Wirklichkeit auf beiden Seiten langfristige und damit unsichere Folgen menschlicher Handlungen abzuwägen sind. Nicht nur der Klimaschutz ist einer Perspektive von mehreren Jahrzehnten unterworfen, obschon sich in der jüngeren Vergangenheit die Anzeichen mehren, dass die befürchteten Änderungen des globalen Klimas eher früher als bislang und eher heftiger als erwartet eintreten werden. Das Gleiche gilt im Kern auch für den Artenschutz, bei dem das einzelne Exemplar in aller Regel nicht um seiner selbst willen geschützt wird, sondern als Teil einer Strategie, dem Artensterben grundsätzlich Einhalt zu gebieten. Zentral ist daher der globale oder - mit Blick auf die Vogelschutzrichtlinie zumindest regionale europäische Erhalt geschützter Populationen (vgl. Art. 2 V-RL, der auf den Erhalt der Bestände (sic!) an Vogelarten zielt). Ein (zu) eng verstandener Individualbezug verstellt den Blick auf die zu lösende Problematik. Beiden Seiten des Spannungsfelds ist zu eigen, dass es um die Abwendung von Risiken geht, die inkrementaler Natur sind, sich langfristig verwirklichen und die für natürlichen Lebensgrundlagen der Menschheit systemrelevant sein dürften, so dass nicht einseitig darauf fokussiert werden darf, dass die Berechnung der Erhöhung des Tötungsrisikos durch die zu erwartenden Vogelkollisionen möglicherweise mit größerer Validität erfolgt, als der Beitrag einer einzelnen Windenergieanlage zum Klimaschutz. Anders mag der Fall zu beurteilen sein, würde die betreffende Art durch den Betrieb einer zu errichtenden bestimmten Windenergieanlage ausgerottet. Dem stünde aber schon das Verschlechterungsverbot des Art. 13 V-RL entgegen.

\section{Verrechnung: gesellschaftlicher positiver Nettonutzen?}

Auch Gellermanns Verrechnungsargument ${ }^{45}$ überzeugt nicht. Stehen wie hier auf beiden Seiten des Spannungsfelds Belange, die durch inkrementale Veränderungen gefördert oder auch beeinträchtigt werden können, so muss sich jede Auslegung, die anhand und mit Blick auf die Lösung eines Einzelfalls entwickelt wird, als verallgemeinerungsfähiger Maßstab rekonstruieren lassen. Daher ist das Gesamtbild der Schutzkonzeption der Vogelschutzrichtlinie und ihr Verhältnis zu anderen öffentlichen Interessen zu betrachten, das zum einen durch ein strenges Schutzregime gekennzeichnet ist, das nur wenige Ausnahmen zu Lasten der geschützten Arten zulässt. Zum anderen ist aber auch die Vogelschutzrichtlinie so konzipiert, dass der Vo-

38) Gellermann, Windkraftnutzung und Schutz europäischer Vogelarten, Möglichkeiten der Erteilung artenschutzrechtlicher Ausnahmen, Rechtswissenschaftliche Stellungnahme, 2020, Stand 11.10.2020. abrufbar unter https://www.naturschutz-initiative.de/images/PDF2020/2020GutachtenProfGellermann.pdf, S. 9

39) Gellermann, Windkraftnutzung und Schutz europäischer Vogelarten, Möglichkeiten der Erteilung artenschutzrechtlicher Ausnahmen, Rechtswissenschaftliche Stellungnahme, 2020, Stand 11.10.2020, abrufbar unter https://www.naturschutz-initiative.de/images/PDF2020/2020GutachtenProfGellermann.pdf, S. 9.

40) So wohl Gellermann, Windkraftnutzung und Schutz europäischer Vogelarten, Möglichkeiten der Erteilung artenschutzrechtlicher Ausnahmen, Rechtswissenschaftliche Stellungnahme, 2020 , Stand 11.10.2020, abrufbar unter https://www.naturschutzinitiative.de/images/PDF2020/2020GutachtenProfGellermann.pdf, S. 9

41) Gellermann, Windkraftnutzung und Schutz europäischer Vogelarten, Möglichkeiten der Erteilung artenschutzrechtlicher Ausnahmen, Rechtswissenschaftliche Stellungnahme, 2020, Stand 11. 10.2020, abrufbar unter https://www.naturschutz-initiative.de/images/PDF2020/2020GutachtenProfGellermann.pdf, S. 9

42) EuGH, Urt. v. 28. 2. 1991 - C-57/89, Slg. 1991, I-883, Rdnr. 25 f. - Leybucht.

43) Gatz, Windenergieanlagen in der Verwaltungs- und Gerichtspraxis, 3. Aufl. 2019, Rdnr. 297, 263.

44) Gatz, Windenergieanlagen in der Verwaltungs- und Gerichtspraxis, 3. Aufl. 2019, Rdnr. 297, 263.

45) Gellermann, Windkraftnutzung und Schutz europäischer Vogelarten, Möglichkeiten der Erteilung artenschutzrechtlicher Ausnahmen, Rechtswissenschaftliche Stellungnahme, 2020, Stand 11.10.2020, abrufbar unter https://www.naturschutz-initiative.de/images/PDF2020/2020GutachtenProfGellermann.pdf, S. 9. 
gelschutz durchaus in bestimmten Fällen gegenüber anderen öffentlichen Interessen zurücktreten kann, darf oder sogar muss, wie sich insbesondere an den Tatbeständen des Art. 9 Abs. 1 V-RL zeigt. Eine „Verrechnung“ von Vorund Nachteilen ist daher als solche weder in der Sache noch nach dem Konzept der Vogelschutzrichtlinie vermeidbar.

Die Offenheit der Vorschrift gegenüber gegenläufigen beachtlichen Interessen wird auch dadurch unterstrichen, dass die Bestimmung der Umsetzung von Art. 9 des Berner Übereinkommens dient, nach dem sich Ausnahmen auch ,im Interesse der öffentlichen Gesundheit und Sicherheit ... oder anderer (Hervorhebung E. H.) vorrangiger öffentlicher Belange" rechtfertigen lassen. Dies spricht dafür, dass zwar nicht beliebige Belange zur Beschränkung des Schutzkonzepts herangezogen werden dürfen. ${ }^{46}$ Ein mögliches $\mathrm{Zu}-$ rücktreten gegenüber bestimmten, hinreichend gewichtigen öffentlichen Interessen gehört jedoch zur Substanz der Regelungen. Insgesamt kommt es für die Wirksamkeit des auf den Erhalt der Bestände gerichteten Konzepts mithin darauf an, dass die Ausnahmen hinsichtlich ihrer einzelnen Tatbestände und bezüglich der Zahl der Tötungen oder Beeinträchtigungen auf lange Sicht das Hauptziel aus Art. 2 V-RL nicht gefährden.

Das leistet eine den Klimaschutz einschließende Auslegung der "Gesundheit“ im Sinne von Art. 9 Abs. 1 V-RL, da neben der spezifischen Engführung des Tatbestands auf die Förderung von Klimaschutzbelangen auch weitere und für den Bau von Windenergieanlagen bedeutsame Sicherungen greifen, die zu einer Begrenzung des Tötungsrisikos führen. Dazu rechnet schon das aus Gründen der VerhältnismäBigkeit geltende Gebot, das signifikante Tötungsrisiko mit Vermeidungsmaßnahmen zu minimieren und das verbleibende Risiko nur dann zu akzeptieren, wenn zumutbare Alternativen nicht vorhanden sind. Art. 9 Abs. $1 \mathrm{~V}$-RL bringt das durch die Maßgabe zum Ausdruck, dass Ausnahmen nur dann erteilt werden dürfen, wenn es keine andere zufriedenstellende Lösung gibt. Das BNatSchG enthält der Sache nach dieselbe Regelung in $₫ 44$ Abs. $5 \mathrm{Nr}$. 1 und $₫ 45$ Abs. 7 S. 2. Letztgenannte Norm stellt darüber hinaus noch die Anforderung, dass sich der Erhaltungszustand der betroffenen Populationen nicht verschlechtern darf, soweit nicht Artikel 16 Absatz 1 der FFH-Richtlinie 92/43/EWG weitergehende Anforderungen enthält. Eine weitere Eingrenzung des Tötungsrisikos erfolgt indirekt über die Bestimmungen des gebietsbezogenen Naturschutzes, die der Errichtung von Anlagen ebenfalls entgegenstehen können. ${ }^{47}$ Schließlich können naturschutzrechtliche Kompensationsmaßnahmen zu einem gewissen Ausgleich (\$6 Abs. 4 FFH-RL, $\$ \$ 13$ Abs. 1, 15 Abs. 2 BNatSchG) und damit zu einem kleineren Nettoverlust an geschützten Tieren beitragen.

Eine Auslegung wie die des VG Gießen, die den Tatbestand der Gesundheit in Art. 9 Abs. 1 V-RL nicht in Erwägung zieht und infolgedessen im deutschen Recht keine entsprechenden Möglichkeiten sucht, dem Interesse am Ausbau der Erneuerbaren Energien im Rahmen der Anwendung der Vogelschutzrichtlinie Berücksichtigung zu verschaffen, verkennt im Ergebnis, dass die Vogelschutzrichtlinie keinen einseitigen und kategorischen Vorrang der Belange des Artenschutzes enthält, sondern ausweislich der genannten Bestimmungen in Art. 2 V-RL und der Ausnahmetatbestände des Art. 9 V-RL der Abwägung mit gegenläufigen Interessen durchaus Raum gibt. ${ }^{48}$ Die Rechtsprechung des EuGH respektiert diese Grundkonzeption. ${ }^{49}$

Das bedeutet allerdings nicht, dass beliebige Gründe, die dem Rechtsanwender bedeutsam erscheinen können, als Rechtfertigungstatbestand in Betracht kommen. Teile der Literatur schießen über die Bestimmungen der Vogelschutzrichtlinie deutlich hinaus, wenn sie fordern, bei richtiger Interpretation könne eine Rechtfertigung auch über Gründe sozialer und wirtschaftlicher Art gelingen. ${ }^{50}$ Die Interpretation wäre nur dann richtig, wenn es bei den Ausnahmetatbeständen der Vogelschutzrichtlinie bleibt und keine darüber hinausgehenden als zulässig erachtet werden. Das ist aber ersichtlich nicht die Zielrichtung der genannten Stimmen in der Literatur, die für eine Harmonisierung von FFH-Richtlinie und Vogelschutzrichtlinie plädieren. Eine solche Interpretation des BNatSchG wäre nicht unionsrechtskonform. ${ }^{51}$

\section{Gestaltungsspielräume der Mitgliedstaaten}

Die Auslegung von Art. 9 Abs. 1 V-RL steht mit den vorstehenden Überlegungen im Schnittfeld von Artenschutzrecht und Klimaschutzrecht, dessen Spannungen nur dann durch adäquate Einzelfallentscheidungen aufgelöst oder zumindest gemindert werden können, wenn man schließlich berücksichtigt, dass das europäische Sekundärrecht in Umsetzung völkerrechtlicher Verpflichtungen aus dem $\mathrm{Pa}-$ riser Übereinkommen festlegt ${ }^{52}$, welche Treibhausgas-Reduzierungen durch die Mitgliedstaaten zu erbringen sind ${ }^{53}$, und dabei gleichzeitig den Mitgliedstaaten Spielräume einräumt, auf welchem Weg sie diese erreichen wollen. ${ }^{54}$ Es ist auch zu erwarten, dass dieser Ansatz in der EU für absehbare Zeit weiter verfolgt werden wird. ${ }^{55}$

46) Gellermann, Windkraftnutzung und Schutz europäischer Vogelarten, Möglichkeiten der Erteilung artenschutzrechtlicher Ausnahmen, Rechtswissenschaftliche Stellungnahme, 2020, Stand 11.10.2020, abrufbar unter https://www.naturschutz-initiative.de/images/PDF2020/2020GutachtenProfGellermann.pdf, S. 12, hält daher eine enge Auslegung des Tatbestands der Sicherheit erforderlich, da nur dann gewährleistet sei, dass nur Gründe herangezogen würden, die ihrem Gewicht nach den ,,zwingenden Gründen des überwiegenden öffentlichen Interesses“ entsprächen.

47) Vgl. Art. 3-11 der Richtlinie 92/43/EWG des Rates vom 21.5. 1992 zur Erhaltung der natürlichen Lebensräume sowie der wildlebenden Tiere und Pflanzen (FFH-RL), ABl. 1992 L 206, S. 7, 10-12, sowie $\$ \$ 20-36$ BNatSchG.

48) So schon Dolde, NVwZ 2007, 7, 11; Lau/Steeck, NuR 2008, 386, $391 \mathrm{f}$.

49) EuGH, Urt. v. 16.10.2003 - C-182/02, Slg. 2003, I-12105, Rdnr. 11.

50) Müller-Mitschke, NuR 2015, 741, 744; de Witt/Geismann, in: de Witt (Hrsg.), Artenschutzrechtliche Verbote in der Fachplanung, Ein Leitfaden für die Praxis zum Bundesnaturschutzgesetz, Verwaltungsrecht für die Praxis Bd. 1, 2. Aufl. 2013, Rdnr. 45; Kautz, NuR 2007, 234, 239; Bick/Wulfert, NuR 2020, 250.

51) Dazu nachstehend 3.1, 3.3.

52) United Nations, Framework Convention on Climate Change, Conference of the Parties, Twenty-first session, Paris, 30 November to 11 December 2015, Agenda item 4(b), Durban Platform for Enhanced Action (decision 1/CP.17), Adoption of a protocol, another legal instrument, or an agreed outcome with legal force under the Convention applicable to all Parties, Adoption of the Paris Agreement, Proposal by the President, Draft decision -/CP.21, FCCC/CP/2015/L.9/Rev.1, 12.12.2015, Stand 21.10.2020, abrufbar unter https://unfccc.int/resource/docs/ 2015/cop21/eng/109r01.pdf, S. 2.

53) Für Deutschland sind das 38 Prozent für das Jahr 2030 gegenüber dem Jahr 2005, s. Art. 4 Abs. 1, Anhang I der Verordnung (EU) 2018/842 des Europäischen Parlaments und des Rates vom 30.5. 2018 zur Festlegung verbindlicher nationaler Jahresziele für die Reduzierung der Treibhausgasemissionen im Zeitraum 2021 bis 2030 als Beitrag zu Klimaschutzmaßnahmen zwecks Erfüllung der Verpflichtungen aus dem Übereinkommen von Paris sowie zur Änderung der Verordnung (EU) Nr. 525/2013, AB1. 2018 L 156, S. 26, 33 f., 39.

54) Siehe insbesondere Richtlinie 2009/28/EG des Europäischen Parlaments und des Rates vom 23.4.2009 zur Förderung der Nutzung von Energie aus erneuerbaren Quellen und zur Änderung und anschließenden Aufhebung der Richtlinien 2001/77/EG und 2003/30/EG, ABl. 2009 L 140, S. 16 ff.; Richtlinie 2001/77/ EG des Europäischen Parlaments und des Rates vom 27.9.2001 zur Förderung der Stromerzeugung aus erneuerbaren Energiequellen im Elektrizitätsbinnenmarkt, ABl. 2001 L 283, S. 33 ff.

55) Europäische Kommission, Vorschlag für eine Verordnung des Europäischen Parlaments und des Rates zur Schaffung des Rahmens für die Verwirklichung der Klimaneutralität und zur Änderung der Verordnung (EU) 2018/1999 (Europäisches Klimagesetz), $\operatorname{COM}(2020) 80$ final. 
Einem solchem System ist immanent, dass das Regelungsumfeld dem politischen Handlungsraum Grenzen ziehen kann. Dazu rechnet auch die Richtlinie (EU) 2018/2001, mit der die Mitgliedstaaten verpflichtet werden, den Anteil der erneuerbaren Energien am Strommix für das Jahr 2030 auf mindestens 32 Prozent zu erhöhen. ${ }^{56}$ Für die Bundesregierung ist hierfür ein bestimmter Anteil an Windenergieanlagen erforderlich. ${ }^{57}$ Dass aber mit dem VG Gießen gerade eine Auslegung der Vogelschutzrichtlinie, die die Berücksichtigung eines nicht von der Hand zu weisenden gesellschaftlichen Interesses an einem wirksamen Klimaschutz nicht zulässt, eine derartige Limitierung markieren soll, die dann zu einer Plankorrektur auf mitgliedstaatlicher Ebene nötigt ${ }^{58}$ und damit $\mathrm{zu}$ einem verstärkten und möglicherweise an anderer Stelle problematischen Ausbau anderer Energiequellen, erscheint angesichts der tatbestandlichen Offenheit von Art. 9 Abs. 1 V-RL wenig plausibel.

Nimmt man diese Eingrenzungen zusammen, so erscheint die Heranziehung des Tatbestands der Gesundheit in Art. 9 Abs. 1 lit. a) V-RL geeignet, um im Zusammenspiel mit den genannten weiteren Regelungselementen der Vogelschutzrichtlinie und den übrigen Vorgaben des Europarechts die Genehmigung von Windenergieanlagen in Einklang mit artenschutzrechtlichen Bestimmungen zu bringen.

\subsubsection{2 Öffentliche Sicherheit}

\section{(Art. 9 Abs. 1 lit. a 1. Spiegelstrich V-RL)}

Im Ergebnis gilt das auch für den Tatbestand der öffentlichen Sicherheit. Der Begriff der öffentlichen Sicherheit wird vom EuGH - allerdings in anderem Kontext - als erfüllt angesehen, wenn eine tatsächliche und hinreichend schwere Gefährdung vorliegt, die ein Grundinteresse der Gesellschaft berührt. ${ }^{59}$ Es genüge, dass das in Rede stehende Vorhaben erforderlich sei, um Risiken für die öffentliche Sicherheit zu verringern. ${ }^{60}$ Auch die deutsche Rechtsprechung teilt überwiegend diesen Grundansatz und vertritt die Auffassung, die ,öffentliche Sicherheit“ in Art. 9 Abs. 1 V-RL sei weit auszulegen. ${ }^{61}$ Ein enges polizeirechtliches Verständnis des Begriffs einschließlich der damit einhergehenden Anforderungen an den Nachweis der Ursächlichkeit ${ }^{62}$ ist mit ihm im Kontext der Vogelschutzrichtlinie nicht verbunden und würde im Regelungskontext der sich gegenüberstehenden Richtlinien - Vogelschutz auf der einen, Klimaschutz auf der anderen Seite - nicht gerecht. ${ }^{63}$ In der Folge würde daher die praktische Wirksamkeit des europarechtlichen Rechtsrahmens unterlaufen.

Gegen eine weite Auslegung der „öffentlichen Sicherheit“ führt Gellermann die Überlegung an, dass nur ,andere zwingende Gründe des überwiegenden öffentlichen Interesses“ die Erhöhung des Tötungsrisikos und andere Beeinträchtigungen zu rechtfertigen imstande seien. ${ }^{64}$ Es könnte daher nur ein sehr begrenzter Kreis öffentlicher Belange gemeint sein, die überhaupt von einer hinreichenden Wichtigkeit seien. Der Klimaschutz, der als ein gesellschaftliches Grundinteresse höchsten Ranges unter die öffentliche Sicherheit im Sinne der Rechtsprechung des EuGH gefasst werden kann, genügt angesichts der Brisanz der Lage diesem Ansatz.

Im Falle der Heranziehung polizeirechtlicher Maßstäbe, nach denen eine Gefahr vorliegt, wenn mit hinreichender Wahrscheinlichkeit ein Schaden an einem Rechtsgut zu erwarten ist, wäre die Verringerung des Ausmaßes klimaänderungsbedingter Schäden durch eine einzelne Anlage oder für einen Windpark wegen ihrer inkrementalen Bedeutung nicht nachzuweisen. Wie schon im Zusammenhang mit dem Tatbestand der Gesundheit erläutert, ${ }^{65}$ bewirkt die einzelne Anlage aber nicht nur eine vernachlässigenswerte inkrementale Veränderung, da ihr Wirkungsbeitrag für den Klimaschutz nicht verlässlich beurteilt werden kann, sie ist gleichzeitig von systemischer Bedeutung, da allein die Gesamtheit der Anlagen den beabsichtigten Effekt der Einsparung von Treibhausgasemissionen in relevanter Menge bewir- ken kann. Der Verzicht auf den Bau einer Anlage wegen mangelnden Nachweises eines Zurechnungszusammenhangs zwischen der einzelnen Anlage und der Begrenzung klimawandelbedingter Schäden würde in der Summe der Einzelentscheidungen eine Gefährdung der Zielerreichung im Klimaschutz bedeuten. Anhaltspunkte für eine so weitreichende Konsequenz artenschutzrechtlicher Vorschriften können Art. 9 Abs. 1 V-RL entgegen der Auffassung des VG Gießen nicht entnommen werden.

Vor diesem Hintergrund der sich dramatisch beschleunigenden globalen Erwärmung ${ }^{66}$ dient der Klimaschutz in der Formulierung des EuGH der Sicherung gesellschaftlicher Kerninteressen, die vom bereits angesprochenen Gesundheitsschutz über wirtschaftliche Interessen etwa im Zusammenhang mit der Nahrungsmittelproduktion bis hin zur Sicherung der Befahrbarkeit von Schifffahrtswegen reicht, die Teil der Infrastruktur für die Versorgung der Bevölkerung mit Lebensmitteln und anderen Erzeugnissen sind. ${ }^{67}$

56) Richtlinie (EU) 2018/2001 des Europäischen Parlaments und des Rates vom 11.12.2018 zur Förderung der Nutzung von Energie aus erneuerbaren Quellen, AB1. 2018 L 328, S. 82, 105 (Art. 3 I).

57) Bundesregierung, Klimaschutzprogramm 2030 der Bundesregierung zur Umsetzung des Klimaschutzplans 2050, 2019, Stand 10.10.2020, abrufbar unter https://www.bundesregierung.de/resource/blob/ 975226/1679914/e01d6bd855f09bf05cf7498e06d0a3ff./2019-1009-klima-massnahmen-data.pdf?download=1, S. 39.

58) Erinnert sei an das Klimaschutzprogramm der Bundesregierung, Klimaschutzprogramm 2030 der Bundesregierung zur Umsetzung des Klimaschutzplans 2050, 2019, Stand 10.10.2020, abrufbar unter https://www.bundesregierung.de/resource/blob/ 975226/1679914/e01d6bd855f09bf05cf7498e06d0a3ff./201910-09-klima-massnahmen-data.pdf?download=1, S. 39.

59) EuGH, Urt. v. 14.3.2000 - C-54/99, Slg. 2000, I-1335, Rdnr. 17; EuGH, Urt. v. 4. 6.2002 - C-503/99, Slg. 2002, I-4809, Rdnr. 47; EuGH, Urt. v. 8.11.2012 - C-244/11, ECLI:EU:C:2012:694, Rdnr. 67.

60) EuGH, Urt. v. 10.7.1984 - C-72/83, Slg. 1984, 2727, Rdnr. 41 - Campus Oil Limited.

61) VGH München, Urt. v. 19.2.2014 - 8 A 11.40040, juris Rdnr. 849; VGH Kassel, Urt. v. 21.8.2009-11 C 318/08.T, juris Rdnr. 771 (,sehr weit zu verstehen“), bestätigt durch BVerwG, Beschl. v. 14. 4. 2011 - 4 B 77/09, juris Rdnr. 82; OVG Koblenz, Urt. v. 8.11.2007 - 8 C 11523/06, juris Rdnr. 199, bestätigt durch BVerwG, Beschl. v. 17.7.2008 - 9 B 15/08, NVwZ 2008, S. 1115

62) So wohl bezüglich von $₫ 45$ Abs. 7 S. 1 Nr. 4 5. Alt. („Umwelt“") BNatSchG Gatz, Windenergieanlagen in der Verwaltungs- und Gerichtspraxis, 3. Aufl. 2019, Rdnr. 297, 263; Lau, in: Frenz/ Müggenborg, Bundesnaturschutzgesetz, Kommentar, 2. Aufl. 2016, \45 Rdnr. 17; deren Auffassung im Zusammenhang mit \45 Abs. 7 S. 1 Nr. 4 BNatSchG (,Umwelt“) ablehnend Sailer, Gesetzgeberische Handlungsmöglichkeiten zur Weiterentwicklung der artenschutzrechtlichen Ausnahmeregelung, Hintergrundpapier, Würzburger Berichte zum Umweltenergierecht, Nr. 49 v. 11.3.2020, Stand 10.10.2020, abrufbar unter https:// stiftung-umweltenergierecht.de/wp-content/uploads/2020/03/ Stiftung_Umweltenergierecht_WueBerichte_49_Artenschutzrechtliche_Ausnahme_2020-03-11.pdf, S. 9.

63) Unzutreffend daher Gatz, Windenergieanlagen in der Verwaltungs- und Gerichtspraxis, 3. Aufl. 2019, Rdnr. 297, 263; Lau, in: Frenz/Müggenborg, Bundesnaturschutzgesetz, Kommentar, 2. Aufl. 2016, $\$ 45$ Rdnr. 17.

64) Gellermann, Windkraftnutzung und Schutz europäischer Vogelarten, Möglichkeiten der Erteilung artenschutzrechtlicher Ausnahmen, Rechtswissenschaftliche Stellungnahme, 2020, Stand 11.10.2020, abrufbar unter https://www.naturschutz-initiative.de/ images/PDF2020/2020GutachtenProfGellermann.pdf, S. 12.

65) S. o. 2.2.2.1.1.

66) Slater/Hogg/Mottram, 10 Nat. Clim. Chang. 2020, 879 ff., Stand 11.10.2020, abrufbar unter https://doi.org/10.1038/s41558020-0893-y.

67) Der Spiegel, NATO-Generalsekretär Stoltenberg: „Der Klimawandel bedroht unsere Sicherheit“, Spiegel Online v. 27.9.2020, mit Hinweis auf einen Gastbeitrag Stoltenbergs in der Welt am Sonntag v. 27.9.2020. 
Gellermann verkennt hingegen die Problematik, wenn er alleine auf die Risiken für die Energieversorgung abstellt, ${ }^{68}$ die möglicherweise auch ohne Verletzung artenschutzrechtlicher Tötungsverbote gewährleistet werden könnte. Zur Begründung verweist er vor allem auf das Urteil des EuGH vom 29. Juli 2019 in der Rechtssache C-411/17 Wallonie. ${ }^{69}$ Der Gerichtshof hatte über die Frage zu befinden gehabt, ob die Überholung und anschließende Wiederinbetriebnahme von Kernkraftwerken mit der Stromversorgungssicherheit eines Mitgliedstaates gerechtfertigt werden könnte, obwohl es um ein Gebiet ging, das einen prioritären natürlichen Lebensraumtyp und/oder eine prioritäre Art einschloss. Für solche Gebiete legt Art. 6 Abs. 4 UA 2 FFH-Richtlinie fest, dass nur Erwägungen im Zusammenhang mit der Gesundheit des Menschen und der öffentlichen Sicherheit oder im Zusammenhang mit maßgeblichen günstigen Auswirkungen für die Umwelt geltend gemacht werden können. Die Richter befanden, nur die Notwendigkeit der Abwendung einer tatsächlichen und schwerwiegenden Gefahr, dass die Stromversorgung des betreffenden Mitgliedstaats unterbrochen wird, unter Umständen wie denen des Ausgangsverfahrens einen Grund der öffentlichen Sicherheit im Sinne dieser Vorschrift darstellen könne. ${ }^{70}$

Trotz der Ähnlichkeiten in der Begriffsbildung (,öffentliche Sicherheit") ist daraus für die Auslegung von Art. 9 Abs. $1 \mathrm{~V}-\mathrm{RL}$ wenig zu gewinnen. Das Urteil betrifft die FFH-Richtlinie, nicht die Vogelschutzrichtlinie. Nimmt man den Ansatz des Gerichtshofs zum Ausgangspunkt, nach welcher die öffentliche Sicherheit dann berührt ist, wenn Grundinteressen der Gesellschaft betroffen sind, dann muss bei einer Windenergieanlage, die in ein nationales Konzept zur Erreichung der Klimaschutzziele eingebunden ist, dieser Tatbestand für Art. 9 Abs. $1 \mathrm{~V}-\mathrm{RL}$ und auch bei der Genehmigung einer einzelnen Anlage bejaht werden können.

\subsubsection{Abwendung erheblicher Schäden an Kulturen, Viehbeständen, Wäldern, Fischereigebieten und Gewässern (3. Spiegelstrich)}

Der dritte Spiegelstrich von Art. 9 Abs. 1 V-RL erfasst die Abwendung erheblicher Schäden an Kulturen, Viehbeständen, Wäldern, Fischereigebieten und Gewässern. Das Schutzgut dieses Rechtfertigungstatbestands ist mithin, etwas verkürzt gesagt, die Landwirtschaft im weiteren Sinn. Bezogen auf die artenschutzrechtliche Rechtfertigung des Baus und Betriebs von Windenergieanlagen bedeutet dies, dass auf die Schäden zu blicken ist, die an den genannten Schutzgütern eintreten würden, bliebe man untätig. Da der Klimawandel absehbar mit einer erhöhten $\mathrm{CO}_{2}$-Konzentration der Luft verbunden ist und dies Auswirkungen auf das Pflanzenwachstum hat, ${ }^{71}$ sind die erforderlichen ursächlichen Zusammenhänge und Risiken dem Grunde nach bekannt. Daher kann es nicht erstaunen, wenn schon seit einiger Zeit landwirtschaftliche Anpassungsstrategien entwickelt werden, um die Schäden möglichst klein zu halten. ${ }^{72}$

Sieht man also von den zunächst mit Blick auf Windenergieanlagen und den Vogelschutz etwas weniger betroffenen Fischereigebieten und Gewässern ab, ist eine Maßnahme, die zur Abschwächung der anthropogen verursachten Erderwärmung getroffen wird, auch eine Maßnahme zur Abwendung erheblicher Schäden an den Schutzgütern des dritten Spiegelstrichs. Dient die Errichtung einer Windenergieanlage der Verwirklichung eines belastbaren Klimaschutzkonzepts im Sinne von Art. 7 des Pariser Übereinkommens, so kann hierfür zur Rechtfertigung auch auf Art. 9 Abs. 1 lit. a) 3. Spiegelstrich zurückgegriffen werden.

\subsubsection{Schutz der Pflanzen- und Tierwelt (4. Spiegelstrich)}

Schließlich können auch Maßnahmen zum Schutz der Pflanzen- und Tierwelt ergriffen werden, selbst wenn dies ein Tötungsverbot nach Art. 5 V-RL verletzt. Damit geht der 4. Spiegelstrich über die Tatbestände des 3. Spiegelstrichs hinaus, indem die Pflanzen- und Tierwelt auch dann geschützt wird, wenn sie nicht einer kommerziellen landwirtschaftlichen Nutzung unterliegt. Die Schäden, um die es im Zusammenhang mit dem Klimawandel geht, sind allerdings dieselben. Sie rechtfertigen daher im selben Umfang wie die drohenden Schäden für die Landwirtschaft Ausnahmen zu Lasten des Artenschutzes.

\subsubsection{Vernünftige Nutzung bestimmter Vogelarten (lit. c)}

Es ist auch zur Diskussion gestellt worden, die windenergiebedingten Kollateralschäden als vernünftige Nutzung europäischer Vogelarten (lit. c) mit der Folge zu interpretieren, dass ein Ausnahmetatbestand des Art. 9 Abs. 1 V-RL erfüllt wäre. ${ }^{73}$ Schon der Wortsinn dürfte einer solchen Auslegung entgegenstehen. Buchstabe c) bezieht sich auf Handlungen, die darauf zielen, Tiere zu nutzen. Ein solches Moment der Absicht ist bei der Tötung, die trotz aller Schutzmaßnahmen nur eine unvermeidliche Nebenfolge des Betriebs einer Windenergieanlage darstellt, nicht zu erkennen, selbst wenn man mit dem EuGH akzeptiert, dass die „Entnahme“ (als ,,vernünftige Nutzung“) im Sinne von Art. 16 Abs. 1 lit. e) FFH-RL auch die Tötung einschließt. ${ }^{74}$ Die vernünftige Nutzung der Vögel scheidet mithin als Rechtfertigungsgrund für die Erhöhung des Tötungsrisikos wildlebender Vogelarten aus, selbst wenn es sich um ,geringe Mengen“ handelt. ${ }^{75}$

\section{Konsequenzen für das deutsche Recht}

Wie vorstehend dargelegt, bietet das Europarecht alles in allem einen leistungsfähigen Rahmen für den Ausgleich zwischen den Belangen des Naturschutzes und denen des Klimaschutzes. Wer aber dem VG Gießen aber in der europarechtlichen Bewertung folgt und annimmt, die Vogelschutzrichtlinie enthalte für die Erteilung einer Genehmigung für eine Windenergieanlage im Falle der signifikanten Erhöhung des Tötungsrisikos für geschützte Vögel keine Möglichkeit zur Erteilung einer Ausnahme, muss konsequenterweise in Kauf nehmen, dass auch das deutsche Recht keine entsprechende Option enthalten kann.

68) Gellermann, Windkraftnutzung und Schutz europäischer Vogelarten, Möglichkeiten der Erteilung artenschutzrechtlicher Ausnahmen, Rechtswissenschaftliche Stellungnahme, 2020, Stand 11.10.2020, abrufbar unter https://www.naturschutz-initiative.de/ images/PDF2020/2020GutachtenProfGellermann.pdf, S. 13f.

69) EuGH, Urt. v. 29.7.2019 - C-411/17, ECLI:EU:C:2019:622 Wallonie

70) EuGH, Urt. v. 29.7.2019 - C-411/17, ECLI:EU:C:2019:622, Rdnr. 159 - Wallonie.

71) Brienen/Caldwell/Duchesne et al., 11 Nat Commun 2020, 4241 , Stand 11.10.2020, abrufbar unter https://doi.org/10.1038/ s41467-020-17966-z.

72) Verband der Landwirtschaftskammern, Klimawandel und Landwirtschaft, Anpassungsstrategien im Ackerbau, 2019, Stand 11.10.2020, abrufbar unter http://www.landwirtschaftskammern.de/pdf/klimawandel.pdf, S. $5 \mathrm{ff}$.

73) VGH München, Urt. v. 19.2.2014 - 8 A 11.40040, juris Rdnr. 851; Sailer, Gesetzgeberische Handlungsmöglichkeiten zur Weiterentwicklung der artenschutzrechtlichen Ausnahmeregelung, Hintergrundpapier, Würzburger Berichte zum Umweltenergierecht, Nr. 49 v. 11.3.2020, Stand 10.10.2020, abrufbar unter https://stiftung-umweltenergierecht.de/wp-content/ uploads/2020/03/Stiftung_Umweltenergierecht_WueBerichte_ 49_Artenschutzrechtliche_Ausnahme_2020-03-11.pdf, S. 7.

74) $\mathrm{EuGH}$, Urt. v. 10.10.2019 - C-674/17, ECLI:EU:C:2019:851, Rdnr. 32.

75) Darin sieht Gellermann ein weiteres Hindernis, s. Gellermann, Windkraftnutzung und Schutz europäischer Vogelarten, Möglichkeiten der Erteilung artenschutzrechtlicher Ausnahmen, Rechtswissenschaftliche Stellungnahme, 2020, Stand 11.10.2020, abrufbar unter https://www.naturschutz-initiative.de/images/ PDF2020/2020GutachtenProfGellermann.pdf, S. $18 \mathrm{f}$. 


\subsection{Ungeschriebener Rechtfertigungsgrund in der Umsetzung durch das BNatSchG?}

Dieses Ergebnis ließe sich nur vermeiden, wenn man in das BNatSchG einen ungeschriebenen Rechtfertigungsgrund hineinlesen würde, der in Anerkennung des öffentlichen Interesses am Ausbau Erneuerbarer Energie im Allgemeinen und der Windenergie an Land im Besonderen die Ausnahmeerteilung gestattet. Ein solcher Vorschlag ist bislang, soweit ersichtlich, jedenfalls noch nicht ausdrücklich gemacht worden. Das kann auch nicht verwundern, da er unter der Annahme, dass sich Art. 9 Abs. 1 V-RL gegen eine Ergänzung um ungeschriebene Ausnahmetatbestände sperrt, gegen den Vorrang des Europarechts verstieße.

Wer hingegen die Auffassung vertritt, eine derartige Ausweitung und/oder Harmonisierung der Vogelschutzrichtlinie auf die in der FFH-Richtlinie enthaltenen Ausnahmegründe sei europarechtlich zulässig, müsste im Bundesnaturschutzgesetz nach einer Umsetzungsvorschrift Ausschau halten. Dafür käme dann $₫ 45$ Abs. 7 S. 1 Nr. 5 BNatSchG in Betracht, die zwingenden Gründe des überwiegenden öffentlichen Interesses einschließlich solcher sozialer und wirtschaftlicher Art. Windenergieanlagen lieBen sich unter einen so weit gefassten Ausnahmetatbestand durchaus subsumieren, der jedoch mit der Rechtsprechung des EuGH nicht unbesehen auf europäische Vogelarten angewendet werden darf, sondern zur Frage führt, ob sich die Vorschrift unionsrechtskonform auslegen lässt (s. u. 3.3).

\subsection{Rechtfertigung über die Gesundheit} und die öffentliche Sicherheit, $\int 45$ Abs. 7 S. 1 Nr. 4 BNatSchG

Mit den Überlegungen unter 2. ist der Weg über eine richtlinienkonforme Auslegung des $₫ 45$ Abs. 7 S. 1 Nr. 5 BNatSchG für den Ausbau der Windenergie allerdings gar nicht erforderlich. So lässt sich ein Verstoß gegen das Tötungsverbot der Vogelschutzrichtlinie infolge des Betriebs von Windenergieanlagen europarechtlich auf die Tatbestände der Gesundheit und der öffentlichen Sicherheit stützen. Da diese Gründe mit $\$ 45$ Abs. 7 S. 1 Nr. 4 BNatSchG Eingang in das deutsche Naturschutzrecht gefunden haben, bieten sie die Möglichkeit, entsprechende Ausnahmen zu erteilen.

Eine wortwörtliche Entsprechung der europarechtlich vorgegebenen Ausnahmegründe aus Art. 9 Abs. 1 lit. a) 3. und 4. Spiegelstrich V-RL (zur Abwendung erheblicher Schäden an Kulturen, Viehbeständen, Wäldern, Fischereigebieten und Gewässern, und zum Schutz der Pflanzenund Tierwelt) findet man hingegen im BNatSchG nicht. \45 Abs. 7 S. 1 Nr. 4 5. Alt. BNatSchG gestattet jedoch die Ausnahmeerteilung auch im Falle, ,der maßgeblich günstigen Auswirkungen auf die Umwelt". Die genannten Spiegelstriche in Art. 9 Abs. 1 V-RL beziehen sich auf Gegenstände, die sich unter den Begriff der „Umwelt“ subsumieren lassen könnten. Möglicherweise wollte der deutsche Umsetzungsgesetzgeber sogar diese Tatbestände mit Hilfe des Begriffs der „Umwelt“ im Sinne einer Zusammenfassung ins deutsche Recht transformieren, nachdem der EuGH im Leybucht-Urteil diesen Weg vorgezeichnet hatte. ${ }^{76}$ Jedenfalls ließe sich die Vorschrift des $\$ 45$ Abs. 7 S. 1 Nr. 4 5. Alt. BNatSchG unionsrechtskonform interpretieren. Das VG Gießen greift daher mit seiner Argumentation zu kurz, der Tatbestand der „Umwelt“ sei in der Vogelschutzrichtlinie nicht wiederzufinden, weshalb insoweit kein Rückgriff auf das BNatSchG erfolgen könne. ${ }^{77}$

\subsection{Richtlinienkonforme Auslegung von $\int 45$ Abs. 7 S. 1 Nr. 5 BNatSchG?}

Wie soeben erläutert, benötigt man den Ausnahmetatbestand der sonstigen zwingenden Gründe des überwiegenden öffentlichen Interesses einschließlich sozialer und wirtschaftlicher Art für den klimapolitisch induzierten Ausbau der Windenergie nach zutreffender Auffassung nicht, da sich andere passende Tatbestände des Art. 9 Abs. 1 V-RL zum Teil sogar wortwörtlich im BNatSchG wiederfinden. Hält man wie das VG Gießen diesen Weg über die Gesundheit, die öffentliche Sicherheit oder die Umwelt hingegen nicht für gangbar, müsste man weiter untersuchen, ob nicht eine Rechtfertigung über die zwingenden Gründe des überwiegenden öffentlichen Interesses einschließlich solcher sozialer und wirtschaftlicher Art erfolgen könnte. Das Verwaltungsgericht ist der Auffassung, dass das Urteil des EuGH in der Rechtssache C-192/11 (Kommission/ Polen) diesen Ausweg versperrt, ${ }^{78}$ da der Gerichtshof eine Norm des polnischen Rechts mit Art. 9 V-RL für unvereinbar erklärt hat, die mit $₫ 45$ Abs. 7 S. 1 Nr. 5 BNatSchG zum Teil wörtlich übereinstimmt. Anders als die polnische Norm schränkt die deutsche Vorschrift jedoch ihren Anwendungsbereich durch verschiedene Bezüge auf das Europarecht ein, ${ }^{79}$ indem sie in den Sätzen 2 und 3 verlangt, dass weitergehende Anforderungen aus Art. 16 Abs. 1 und 3 der Richtlinie 92/43/EWG und Artikel 9 Absatz 2 der Richtlinie 2009/147/EG beachtet werden. Darin liegt ein bedeutsamer Unterschied zur polnischen Regelung. ${ }^{80} \mathrm{Al}$ lerdings beantwortet er nicht die Frage, ob $₫ 45$ Abs. 7 S. 1 Nr. 5 BNatSchG mit Art. 9 Abs. $1 \mathrm{~V}-\mathrm{RL}$ vollkommen in Einklang steht.

Das dürfte mit gravierenden Folgen zu verneinen sein. ${ }^{81}$ Solange $₫ 45$ Abs. 7 S. 1 Nr. 5 BNatSchG - wie von der Bundesregierung intendiert ${ }^{82}-$ auch europäische Vogelarten betrifft und keine Einschränkung der ,zwingenden Gründe des überwiegenden öffentlichen Interesses einschließlich solcher sozialer und wirtschaftlicher Art" enthält, die dem abgeschlossenen Katalog des Art. 9 Abs. 1 V-RL Geltung verschafft, ist die Norm zu weit gefasst. Sie lässt die Erteilung einer Ausnahme auch in Fällen zu, die unter Art. 9 Abs. $1 \mathrm{~V}$-RL nicht zulässig sein dürften, wie etwa die Bezugnahme auf eine regionale Wirtschaftsförderung, die Schaffung oder Sicherung von Arbeitsplätzen oder weitere strukturpolitische Gründe. Die zum Teil als Beleg des Gegenteils angeführte Rechtsprechung betrifft entweder keine Windenergieanlagen oder argumentiert maßgeblich mit anderen Tatbeständen des $\$ 45$ Abs. 7 BNatSchG. So hatte der Bayerische Verwaltungsgerichtshof in seinem Urteil vom 19.2.2014 zum Flughafen München die artenschutzrechtliche Rechtmäßigkeit der Genehmigungsentscheidung im Wesentlichen mit der Vereinbarkeit der Ausnahmeerteilung unter Heranziehung der Tatbestände der „öffentlichen Sicherheit“ (Art. 9 Abs. 1 lit. a 1. Spiegelstrich V-RL) und den Interessen der Luftfahrt (Art. 9 Abs. 1 lit. a 2. Spiegelstrich V-RL) begründet. ${ }^{83}$ Bezüglich der Umsetzung verwies der Verwaltungsgerichtshof darauf, dass der Tatbestand der öffentlichen Sicherheit im BNatSchG in $\$ 45$ Abs. 7 S. 1 Nr. 4 verankert sei. ${ }^{84}$

76) EuGH, Urt. v. 28.2.1991 - C-57/89, Slg. 1991, I-883, Rdnr. 22 - Leybucht.

77) VG Gießen, Urt. v. 22.1.2020 - 1 K 6019/18.GI, juris Rdnr. 117.

78) VG Gießen, Urt. v. 22.1.2020 - 1 K 6019/18.GI, juris Rdnr. $107 \mathrm{ff}$

79) Karpenstein/Engel, ZUR 2020, 437, 438 r. Sp.

80) Karpenstein/Engel, ZUR 2020, 437, 438 r. Sp.

81) Anders Teile der Rechtsprechung nach dem Polen-Urteil des EuGH v. 26.1.2012 - C-192/11, ECLI:EU:C:2012:44; OVG Berlin-Brandenburg, Beschl. v. 20.2.2020-OVG 11 S 8/20, juris Rdnr. 39; VGH München, Urt. v. 19.2.2014 - 8 A 11.40040, juris Rdnr. 846ff.; VGH Kassel, Beschl. v. 6.1.2020 - 9 B 1876/18, juris Rdnr. 29; im Ergebnis auch VGH Kassel, Beschl. v. 21.2.2019 - 9 B 182/19, juris Rdnr. 16, 34; OVG Lüneburg, Beschl. v. 19.12.2016 - 12 ME 85/16, juris Rdnr. 36.

82) $₫ 45$ Abs. 7 S. 1 Nr. 5 BNatSchG sollte eine Umsetzung für beide Richtlinien (V-RL und FFH-RL) sein, BT-Drs. 16/5100, 2007, S. 13 .

83) VGH München, Urt. v. 19.2.2014-8 A 11.40040, juris Rdnr. 848. 84) VGH München, Urt. v. 19.2.2014-8 A 11.40040, juris Rdnr. 848. 
Die weitere Begründung des Gerichts, der Flughafenausbau diene Interessen, die über das rein Wirtschaftliche hinausgingen und als bedeutende Infrastrukturmaßnahme auch als vernünftige Nutzung bestimmter Vogelarten zu bewerten seien, ${ }^{85}$ trifft erstens nicht die Vereinbarkeit des $\$ 45$ Abs. 7 S. 1 Nr. 5 BNatSchG mit der Vogelschutzrichtlinie und dürfte zweitens mit den vorstehenden Überlegungen nur dann im Ergebnis als zutreffend zu beurteilen sein, wenn man die Argumentation des Verwaltungsgerichtshofs als unionsrechtskonforme Auslegung der zwingenden Gründe mit Blick auf den Ausnahmetatbestand der Sicherheit der Luftfahrt rekonstruiert. Als Beleg für die Anerkennung der generellen Unionsrechtskonformität des $₫ 45$ Abs. 7 S. 1 Nr. 5 BNatSchG lässt sich das Urteil jedenfalls nicht heranziehen.

So darf festgehalten werden, dass $\int 45$ Abs. 7 S. 1 Nr. 5 BNatSchG mit den vorstehenden Überlegungen nicht europarechtskonform ist, soweit sich die Vorschrift auch auf europäische Vogelarten im Sinne der Vogelschutzrichtlinie bezieht. Daran kann auch nichts ändern, dass die genannte Bestimmung des BNatSchG einige einschränkende Elemente der Vogelschutzrichtlinie enthält, so etwa die Anforderungen an die Alternativenwahl und das Populationsverschlechterungsverbot (\$45 Abs. 7 S. 2 BNatSchG). Die noch darüber hinausgehenden Anforderungen in $\$ 45$ Abs. 7 S. 3 BNatSchG, nach denen 16 Absatz 3 der Richtlinie 92/43/ EWG und Artikel 9 Absatz 2 der Richtlinie 2009/147/EG zu beachten sind, gewährleisten gerade nicht, dass die zur Rechtfertigung von Ausnahmen heranzuziehenden Gründe im Anwendungsbereich der Vogelschutzrichtlinie auf diejenigen des Art. 9 Abs. 1 V-RL beschränkt bleiben.

In dieser überschießenden Wirkung des $₫ 45$ Abs. 7 S. 1 Nr. 5 BNatSchG liegt daher nach der hier vertretenen Rechtsauffassung eine Vertragsverletzung. Die EU-Kommission hat diese bislang nicht gerügt, eine entsprechende Verurteilung der Bundesrepublik Deutschland liegt nicht vor. Das würde eine Gelegenheit eröffnen, mit einer Änderung des BNatSchG Klarheit zu schaffen. Solange aber die Gesetzeslage in Deutschland unverändert bestehen bleibt, ist in der Rechtsanwendung nach einer unionsrechtsformen Auslegung von $₫ 45$ Abs. 7 S. 1 Nr. 5 BNatSchG Ausschau zu halten. Diese kann auch gelingen. Die Formulierung der Vorschrift geht über Art. 9 Abs. 1 V-RL hinaus, kann aber durch eine richtlinienkonforme Reduktion auf die Gründe beschränkt werden, die mit der Vogelschutzrichtlinie vereinbar sind. Soweit das in der Genehmigungspraxis geschieht, ist es möglich, einen Verstoß gegen die Vogelschutzrichtlinie zu vermeiden.

Eine korrekte Umsetzung von Art. 9 Abs. $1 \mathrm{~V}-\mathrm{RL}$ ist die bloße Anwendung in den Genehmigungsverfahren aber nicht, da eine Verwaltungspraxis jederzeit modifiziert werden kann und daher den Anforderungen des EuGH an eine rechtssichere Umsetzung nicht genügt. ${ }^{86}$ Erforderlich ist mithin die Begrenzung von $₫ 45$ Abs. 7 S. 1 Nr. 5 BNatSchG auf die Tatbestände des Art. 9 Abs. 1 V-RL. Eine Möglichkeit, dies im deutschen Recht umzusetzen, wäre die Ergänzung von $\ 45$ Abs. 7 S. 1 Nr. 5 S. 3 BNatSchG durch eine Inbezugnahme von Art. 9 Abs. 1 V-RL. Die Norm könnte daher im Ergebnis ungefähr lauten:

,Artikel 16 Absatz 3 der Richtlinie 92/43/EWG und Artikel 9 Absätze 1 und 2 der Richtlinie 2009/147/EG sind zu beachten“.

\section{Fazit und Ausblick}

Es lässt sich wie folgt bilanzieren: Das Urteil des VG GieBen vom 22. Januar 2020 hat einen weiteren Anstoß zur Diskussion über das Verhältnis des Artenschutzrechts zu gegenläufigen öffentlichen Interessen gegeben. Solange insbesondere die Frage, ob der Bau von Windenergieanlagen nicht unter einen der Tatbestände des Art. 9 Abs. 1 V-RL fallen kann, wenn und soweit das Projekt rechtfertigungsbedürftige Tötungen geschützter Vogelarten mit sich bringt, nicht unmittelbar durch den EuGH geklärt wird, muss eine Analyse bei den europarechtlichen Vorgaben ansetzen. Zunächst war daher der Begriff der absichtlichen Tötung zu untersuchen, der in der Rechtsprechung des EuGH für den Anwendungsbereich der FFH-Richtlinie bereits dann erfüllt ist, wenn es zu Tötungen kommt, die sich als unvermeidliche Folge menschlicher Handlungen darstellen und durch die die Mortalität gegenüber dem allgemeinen Lebensrisiko signifikant erhöht wird. Vorbehaltlich einer Übernahme des Vorschlags von Frau Generalanwältin Kokott bleibt es dabei, dass dann, wenn eine Anlage trotz einer rechtfertigungsbedürftigen Tötung gebaut und betrieben werden soll, dies die Vogelschutzrichtlinie erlaubt, daran aber strenge Anforderungen stellt.

Art. 9 Abs. $1 \mathrm{~V}-\mathrm{RL}$ verlangt zunächst, dass es keine andere zufriedenstellende Lösung geben darf (Erforderlichkeit). Auch darf sich der Erhaltungszustand der betroffenen Arten nicht verschlechtern (Art. 13 V-RL). Der materielle Kern der Zulässigkeit der Ausnahme liegt dann weiter darin, dass sie nur mit Hilfe der Gründe, die in dem abgeschlossenen Katalog des Art. 9 Abs. 1 V-RL aufgeführt sind, gelingen kann. Das bedeutet zunächst, dass die Ergänzung der Tatbestände um weitere ungeschriebene Ausnahmetatbestände ausgeschlossen ist.

Im Rahmen des Art. 9 Abs. 1 V-RL gibt es allerdings eine Reihe von Tatbeständen, die für die Erteilung einer Ausnahme zugunsten von Windenergieanlagen herangezogen werden können. Mit den vorstehenden Überlegungen lassen sich Windenergieanlagen mit der Förderung des Gesundheitsschutzes, der öffentlichen Sicherheit, der Landwirtschaft und der Pflanzen- und Tierwelt begründen. Diese Rechtfertigungsgründe finden sich zum Großteil auch im BNatSchG wieder, so dass die naturschutzrechtliche Zulässigkeit derartiger Anlagen unter bestimmten Voraussetzungen durchaus hergestellt werden kann. Diese Voraussetzungen müssen dem strengen Schutzkonzept Rechnung tragen und im Ergebnis zu Tötungszahlen führen, die mit dem Erhaltungsziel der Richtlinie vereinbar sind. Da die Vogelschutzrichtlinie nur in ihrem Regelungsumfeld angemessen bewertet werden kann, gilt insgesamt, dass die signifikante Erhöhung des Risikos für Kollisionsverluste als Kollateralschaden des Betriebs von Windenergieanlagen dann gerechtfertigt ist, wenn

- der gebietsbezogene Naturschutz dies zulässt,

- zumutbare (Standort-)Alternativen nicht gegeben sind (\$ 45 Abs. 7 S. 1 Nr. 5 S. 2 1. Alt. BNatSchG),

- sich der Erhaltungszustand der betroffenen Populationen nicht verschlechtert ( $\$ 45$ Abs. 7 S. 1 Nr. 5 S. 2 2. Alt. BNatSchG),

- ein vollständiger Ausgleich der Eingriffe zulasten betroffener europäischer Vogelarten angeordnet worden ist,

- die Rechtfertigung des Tötungsrisikos über in primärrechtskonformer Auslegung der Vogelschutzrichtlinie öffentliche Sicherheit (1. Spiegelstrich), Abwendung von Gefahren für die Pflanzen- und Tierwelt (4. Spiegelstrich) oder für die Kulturen und Viehbestände (3. Spiegelstrich) in hinreichendem Maß gelingt,

- insgesamt also die Zahl der zu erwartenden toten Tiere gering ist, die Erhaltung der Populationen nicht gefährdet wird und das Eingehen des Risikos zur Erreichung der Klimaschutzziele notwendig ist.

Für die Umsetzung der V-RL ins deutsche Recht bedeutet das, dass $\$ 45$ Abs. 7 S. 1 Nr. 5 BNatSchG eine über-

85) VGH München, Urt. v. 19.2.2014-8 A 11.40040, juris Rdnr. 851 86) EuGH, Urt. v. 6.5.1980 - C-102/79, Slg. 1980, 1473, Rdnr. 11 - Kommission/Belgien; ähnlich EuGH, Urt. v. 15.10.1986 - C-168/85, Slg. 1986, 2945, Rdnr. 13 - Kommission/Italien; EuGH, Urt. v. 30.5. 1991 - C-59/89, Slg. 1991, I-2626, Rdnr. $28-$ Kommission/Deutschland; EuGH, Urt. v. 28.2.1991-C-131/88, Slg. 1991, I-865, Rdnr. 61 - Kommission/Deutschland. 
schießende Wirkung aufweist, die eine Vertragsverletzung darstellt, die nur der Gesetzgeber beseitigen kann. Bis auf weiteres ist die Vorschrift europarechtskonform, also vogelschutzrichtlinienkonform in dem Sinne auszulegen, dass nicht beliebige öffentliche Interessen den Tatbestand zu erfüllen imstande sind, sondern von der Art her nur diejenigen, die die Vogelschutzrichtlinie in Art. 9 Abs. 1 V-RL zulässt, und von der Intensität her als ,,zwingend“ zu bewerten sind, sich also als im strengen Sinn erforderliche Maßnahmen erweisen müssen; die Gründe dürfen daher nicht nur unspezifischer sozialer und wirtschaftlicher Art sein ( $\$ 45$ Abs. 7 S. 1 Nr. 5 BNatSchG), sondern sie müssen den Tatbeständen der V-RL entsprechen. Das wäre mit Hilfe einer richtlinienkonformen Reduktion des BNatSchG zu erreichen.

Open Access. Dieser Artikel wird unter der Creative Commons Namensnennung 4.0 International Lizenz veröffentlicht, welche die
Nutzung, Vervielfältigung, Bearbeitung, Verbreitung und Wiedergabe in jeglichem Medium und Format erlaubt, sofern Sie den/die ursprünglichen Autor(en) und die Quelle ordnungsgemäß nennen, einen Link zur Creative Commons Lizenz beifügen und angeben, ob Änderungen vorgenommen wurden.

Die in diesem Artikel enthaltenen Bilder und sonstiges Drittmaterial unterliegen ebenfalls der genannten Creative Commons Lizenz, sofern sich aus der Abbildungslegende nichts anderes ergibt. Sofern das betreffende Material nicht unter der genannten Creative Commons Lizenz steht und die betreffende Handlung nicht nach gesetzlichen Vorschriften erlaubt ist, ist für die oben aufgeführten Weiterverwendungen des Materials die Einwilligung des jeweiligen Rechteinhabers einzuholen.

Weitere Details zur Lizenz entnehmen Sie bitte der Lizenzinformation auf http://creativecommons.org/licenses/by/4.0/deed.de.

Open Access funding enabled and organized by Projekt DEAL.

\title{
Ein Landwirtschaftsgesetz für Deutschland im Zeichen von Umweltschutz und Biodiversität - Notwendigkeit, Funktion und Leitbild
}

\author{
Detlef Czybulka/Peter Fischer-Hüftle/Ulrich Hampicke/Wolfgang Köck/José Martinez
}

( ) Der/die Autor(en) 2021. Dieser Artikel ist eine Open-Access-Publikation.

Der Deutsche Naturschutzrechtstag (DNRT) hat 2018 in seiner „Leipziger Erklärung“ gefordert, ein nationales Landwirtschaftsgesetz mit Betreiberpflichten für die Betriebe zu schaffen, mit dem der dramatische Rückgang der biologischen Vielfalt in der Agrarlandschaft gestoppt werden soll. Gegen diese Stoßrichtung sind Einwände erhoben worden, u. a. im Hinblick auf europäische Zuständigkeiten und begrenzte Bundesgesetzgebungskompetenzen. Die Autoren möchten aufzeigen, dass das Nebeneinander von europäischem und deutschem Landwirtschaftsrecht im Zeichen der Biodiversität, des Umwelt- und Tierschutzrechts neu geordnet werden kann und muss. Dabei ist der deutsche Gesetzgeber in der Verantwortung und hat für die hier entworfene Konzeption auch die erforderlichen Kompetenzen. Der Beitrag besteht aus zwei Teilen.

Prof. Dr. Detlef Czybulka, Universität Rostock,

Vorsitzender des Deutschen Naturschutzrechtstages e. V.,

Rostock, Deutschland

Peter Fischer-Hüftle, Vors. Richter am VG a. D., Rechtsanwalt, Regensburg, Deutschland

Prof. Dr. Ulrich Hampicke,

ehemals Lehrstuhl für Landschaftsökonomie, Universität Greifswald, Greifswald, Deutschland

Prof. Dr. Wolfgang Köck, Mitglied des Sachverständigenrates für Umweltfragen (SRU),

Leiter des Departments für Umwelt- und Planungsrecht am Helmholtz-Zentrum für Umweltforschung - UFZ,

Leipzig, Deutschland

Prof. Dr. José Martinez,

Geschäftsführender Direktor des Instituts für Landwirtschaftsrecht der Universität Göttingen,

Mitglied des Wissenschaftlichen Beirats für Agrarpolitik,

Ernährung und gesundheitlichen Verbraucherschutz beim BMEL

sowie Zweiter Vorsitzender

der Deutschen Gesellschaft für Agrarrecht,

Göttingen, Deutschland
Die vorliegende Abhandlung befasst sich mit Notwendigkeit, Funktion und Leitbild eines neuen Landwirtschaftsgesetzes. Der demnächst folgende zweite Aufsatz $z^{1}$ widmet sich den erforderlichen materiellen Regelungen und der Finanzierung.

\section{Warum wir ein Landwirtschaftsgesetz brauchen!}

Die Landwirtschaft soll ,nachhaltig, ökologisch verantwortbar, ökonomisch leistungsfähig und regional verankert“ sein und gerade dadurch auch ,einen Beitrag zur Erhaltung lebenswerter ländlicher Räume" leisten, heißt es im (politischen) Leitbild der Bundesregierung für eine zukunftsfähige Landwirtschaft. ${ }^{2}$ Davon ist der Zustand der Landwirtschaft weit entfernt, ganz besonders, wenn auf die ,ökologische Verantwortbarkeit" geschaut wird. Die auf der Hälfte der Fläche Deutschlands ausgeübte industrialisierte und intensive Landwirtschaft ist für den Biodiversitätsverslust in der Kulturlandschaft an erster Stelle verantwortlich. ${ }^{3}$ Der Prozess hat vor mindestens 40 Jahren eingesetzt und beschleunigt sich. Hunderte ,gewöhnliche“ Pflanzenarten (nicht etwa Orchideen), die die Agrarlandschaft seit jeher geprägt hatten, sind auf wenig mehr als fünf Prozent ihrer Population der 1950er Jahre reduziert. ${ }^{4}$

1) Er trägt den Titel „Ein Landwirtschaftsgesetz für Deutschland im Zeichen des Umweltschutzes und der Biodiversität - Zentrale Gesetzesinhalte und Finanzierung" und ist gemeint, wenn nachfolgend auf „Teil 2“ verwiesen wird.

2) Agrarpolitischer Bericht der Bundesregierung 2019, S. $12 \mathrm{ff}$.

3) Nationale Akademie der Wissenschaften (Leopoldina), Artenrückgang in der Agrarlandschaft, Was wissen wir und was können wir tun, 2018

4) Leuschner/Krause/Meyer/Bartels, Strukturwandel im Acker und Grünland Niedersachsens und Schleswig-Holsteins seit 1950. Natur und Landschaft 89, 2014, 386. Weiteres Material u. a. in BfN (Bundesamt für Naturschutz) 2016: Daten zur Natur 2016.; BfN 2017 Agrar-Report 2017, Biologische Vielfalt in der Agrarlandschaft. 